ARTICLE

\title{
Natural antisense transcripts of MIR398 genes suppress microR398 processing and attenuate plant thermotolerance
}

\author{
Yajie Li (iD ${ }^{1,2}$, Xiaorong $\mathrm{Li}^{1,2}$, Jun Yang (iD ${ }^{1 凶} \&$ Yuke He (i) ${ }^{1 凶}$
}

MicroRNAs (miRNAs) and natural antisense transcripts (NATs) control many biological processes and have been broadly applied for genetic manipulation of eukaryotic gene expression. Still unclear, however, are whether and how NATs regulate miRNA production. Here, we report that the cis-NATs of MIR398 genes repress the processing of their pri-miRNAs. Through genome-wide analysis of RNA sequencing data, we identify cis-NATs of MIRNA genes in Arabidopsis and Brassica. In Arabidopsis, MIR398b and MIR398c are coexpressed in vascular tissues with their antisense genes NAT398b and NAT398c, respectively. Knock down of NAT398b and NAT398c promotes miR398 processing, resulting in stronger plant thermotolerance owing to silencing of miR398-targeted genes; in contrast, their overexpression activates NAT398b and NAT398c, causing poorer thermotolerance due to the upregulation of miR398-targeted genes. Unexpectedly, overexpression of MIR398b and MIR398c activates NAT398b and NAT398c. Taken together, these results suggest that NAT398b/c repress miR398 biogenesis and attenuate plant thermotolerance via a regulatory loop.

\footnotetext{
${ }^{1}$ National Key Laboratory of Plant Molecular Genetics, CAS Center for Excellence in Molecular Plant Sciences, Shanghai Institute of Plant Physiology and Ecology, Chinese Academy of Sciences, 200032 Shanghai, China. ${ }^{2}$ University of the Chinese Academy of Sciences, 100049 Beijing, China.

凶email: jyang@cemps.ac.cn; heyk@cemps.ac.cn
} 
M iRNAs (microRNAs) are small RNAs that repress the expression of their target genes at post-transcriptional levels ${ }^{1}$. In plants, appropriate miRNA accumulation depends on the activity of the nuclear proteins HYPONASTIC LEAVES1 (HYL1), DICER-LIKE1 (DCL1), SERRATE (SE), and AGONAUTE1 (AGO1) ${ }^{2-4}$. These proteins are thought to function together to catalyze the processing of pri-miRNAs and premiRNAs ${ }^{5-7}$. Nearly all nonlethal mutants of $D C L 1, H Y L 1$, and $S E$ display multiple morphological, physiological, and biochemical aberrations ${ }^{8}$. Theoretically, the pleiotropic effects of DCL1, HYL1, and $S E$ mutations are attributable to the reduced accumulation of miRNAs and increased expression of miRNA-directed targets ${ }^{8,9}$. Because miRNAs can silence their target genes ${ }^{10}$, MIRNA genes and artificial miRNAs have been broadly used for identification of gene function in plants and for genetic manipulation of agricultural traits for high yield and quality ${ }^{11,12}$. For unknown reasons, however, these MIRNA genes frequently do not work in many plants ${ }^{13}$.

Natural antisense transcripts (NATs), a class of RNAs containing sequences complementary to sense mRNAs, comprise a set of prominent and complex regulatory RNAs. Recent genomic studies using computational prediction methods and experimental identification approaches have revealed a large number of NATs ${ }^{14-17}$. NATs can be divided into cis-NATs, which are transcribed from opposing DNA strands at the same genomic locus, and trans-NATs, which are transcribed from separate genomic loci. Cis-NATs in turn can be categorized into three types: convergent ( $3^{\prime}$-end overlap), divergent ( $5^{\prime}$-end overlap), and enclosed (one transcript encompassing the other) ${ }^{18,19}$. Studies have revealed that cis-NATs can participate in a broad range of regulatory events ${ }^{20}$. Evidence has been uncovered for the involvement of cis-NATs in translation initiation ${ }^{21,22}$, mRNA stability ${ }^{23}$, transcription termination ${ }^{24}$, alternative splicing $^{25,26}$, RNA editing ${ }^{27,28}$, DNA methylation ${ }^{29,30}$, histone methylation 31,32 , small interfering RNA (siRNA)-induced gene silencing ${ }^{33,34}$, and translational enhancement ${ }^{35,36}$. Luo et al. (2009) have shown that antisense transcription is associated with miRNA-targeted mRNAs in Arabidopsis ${ }^{37}$. Even though several cis-NATs are known to take part in gene regulatory events, the role of many cis-NATs is unclear.

Most documented cis-NATs correspond to protein-coding genes, with only a few cis-NATs of noncoding MIRNA genes having been reported $36,38,39$. Our previous study first identified a subset of cis-NATs corresponding to MIRNA genes in Brassica rapa ${ }^{16}$, including BrpMIR398b and BrpMIR398c. miR398, a conserved miRNA that targets $C S D 1, C S D 2$, and $C C S^{40}$, is proposed to be directly linked to cell death ${ }^{41}$ and responses to oxidative stress ${ }^{13}$, heat stress ${ }^{42}$, water deficit ${ }^{43}$, abscisic acid stress ${ }^{44}$, high sucrose ${ }^{45}$, and bacterial infection ${ }^{34}$. Heat stress can injure a broad spectrum of cellular components and adversely affects the distribution and productivity of horticulturally and agriculturally important plants worldwide ${ }^{42,46}$. Heat stress rapidly induces miR398 and reduces transcripts of its target genes CSD1, CSD2, and CCS. Transgenic plants expressing CSD1, CSD2, and CCS are more sensitive to heat stress than wild-type plants. In contrast, $c s d 1, c s d 2$, and $c c s$ mutant plants are more heat tolerant than the wild-type ${ }^{42}$.

In the study reported here, we endeavored to understand the molecular relationship between MIRNA genes and their cisNATs in miRNA processing and gene regulation in response to high temperature. Functional analysis indicated that the processing of pri-miR398b/c is suppressed by their cis-NATs. This finding provides insight into the function of cis-NATs in miRNA-guided gene silencing and should thus facilitate the genetic manipulation of gene expression for thermotolerance in eukaryotes.

\section{Results}

Identification of cis-NATs at MIR398 gene loci of B. rapa and Arabidopsis thaliana. In our previous study, we examined the high-temperature response of $1031 \mathrm{~B}$. rapa cis-NATs, eight of which corresponded to precursors of miRNAs ${ }^{16}$. To explore the genome-wide heat stress response of additional novel cis-NATs of MIRNA genes in B. rapa, we carried out DNA resequencing and RNA sequencing (RNA-seq) of B. rapa ssp. pekinensis 'Bre' (a heading Chinese cabbage) exposed to $42^{\circ} \mathrm{C}$ for 0 and 1 h. In combination with our RNA-seq data and B. rapa mRNA sequences deposited in the Brassica Database (http://brassicadb. $\mathrm{org} / \mathrm{brad} /$ ), we identified 22 cis-NATs that were reversecomplementary to MIRNA genes. These cis-NATs were thus designated as cis-NATs of MIRNA genes in B. rapa (Supplementary Table 1).

Four copies of the MIR398 gene-BrpMIR398a-1, BrpMIR398a-2, BrpMIR398b-1, and BrpMIR398b-2-were identified in the Brassica genome (Fig. 1a). Among them, the miRNA precursors of BrpMIR398b-1 and BrpMIR398b-2 were sense genes that overlapped with cis-NATs of BrpNAT398b-1 (Bra006261) and BrpNAT398b-2 (Bra008752), respectively. Analysis of the RNAseq data revealed that the transcripts of pri-miR398b-2 and BrpNAT398b-2 nearly fully overlapped with each another (Fig. 1a). According to the latest annotations in the Brassica Database, the BrpNAT398b-1 gene encodes a 963-amino-acid protein of a putative polyribonucleotide nucleotidyltransferase. The BrpNAT398b-2 gene encodes a 366-amino-acid protein of unknown function belonging to the Core-2/I-branching beta-1,6$\mathrm{N}$-acetylglucosaminyltransferase family.

Because A. thaliana is a close relative of $B$. rapa, we searched for MIRNA genes and their cis-NATs in Arabidopsis using the same criteria used for B. rapa. Twenty-five cis-NATs homologous to $B$. rapa cis-NATs or reverse-complementary to Arabidopsis MIRNA genes were identified and regarded as cis-NATs of MIRNA genes in A. thaliana (Supplementary Table 1). The Arabidopsis genome contains three copies of the MIR398 gene: MIR398a (AT2G03445), MIR398b (AT5G14545) and MIR398c (AT5G14565) (Fig. 1b). We examined cis-NATs of MIR398b and MIR398c (NAT398b and NAT398c) but failed to identify the cisNAT of MIR398a. NAT398b is predicted to encode a member of the Core-2/I-branching beta-1,6-N-acetylglucosaminyltransferase family and is homologous to BrpNAT398b-2 in B. rapa, with the two genes sharing $74.3 \%$ and $84.4 \%$ nucleic acid and amino acid sequence identities, respectively. A proteome analysis revealed that NAT398c encodes high-affinity nitrate transporter $2.7^{47}$. To obtain full-length transcripts of MIR398b and MIR398c genes and their cis-NATs, we performed a RACE assay and found that the longest transcripts of pri-miR398b, pri-miR398c, NAT398b, and NAT398c were 692, 1941, 1772, and 1917 nt, respectively (Fig. 1b). In addition, we noticed that pri-miR398b nearly full overlapped with NAT398b (Supplementary Fig. 2a), while pri-miR398c partially overlapped with NAT398c (Supplementary Fig. 2b).

We further discovered that MIR398 genes and their cis-NATs are conserved in angiosperms, especially Brassicaceae. All 12 analyzed species in this plant family were found to have MIR398 genes and their cis-NATs. Most of these NAT398 genes encode core-2/I-branching enzyme, nitrate transporter 2.7 or putative polyribonucleotide nucleotidyltransferase, as in $B$. rapa and $A$. thaliana (Supplementary Data 2). These results imply that MIR398 and NAT398 genes are highly conserved in cruciferous plants.

Overexpression of $M I R 398 \mathrm{~b}$ and $M I R 398 \mathrm{c}$ genes fails to elevate miR398 levels. The MIR398 gene family in Arabidopsis comprises MIR398a, MIR398b, and MIR398c, but MIR398a is not 
a
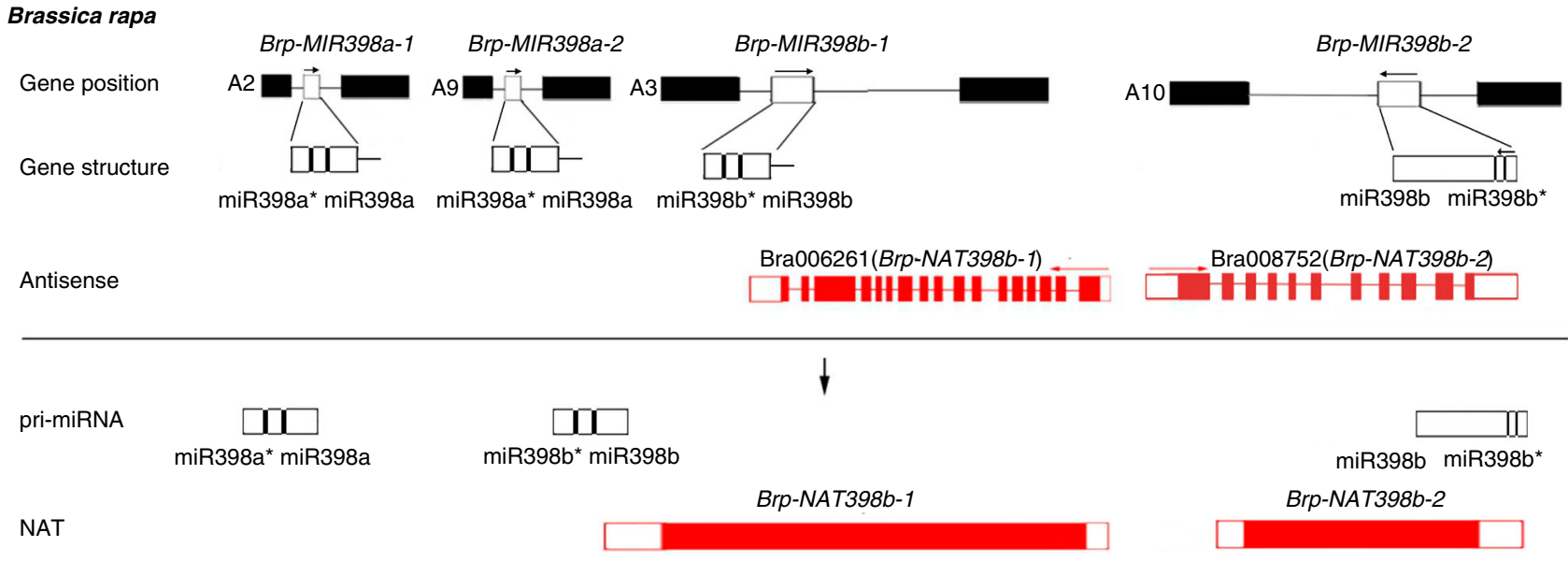

b
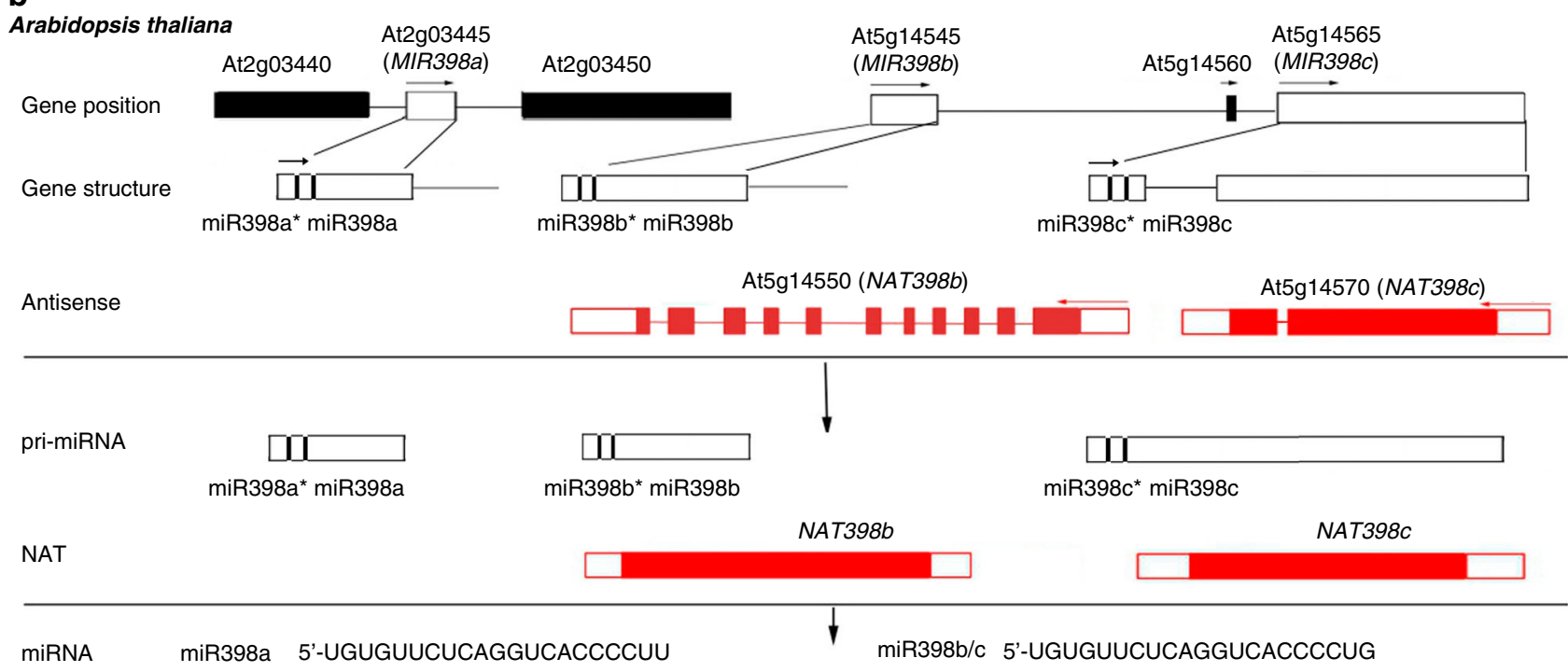

nat-siRNA nat-siR398-1 5'-AACGAGAGCUGUAACAAUGAC

Fig. 1 Schematic positions and structures of MIR398 genes and their cis-NATs in Arabidopsis thaliana and Brassica rapa. a, b Schematic positions and structures of BrpMIR398 genes and their cis-NATs in B. rapa (a) and MIR398 genes and their cis-NATs in A. thaliana (b). Flanking genes, MIRNA genes and exons are enclosed in black, white and red boxes, respectively. Solid lines indicate introns and intergenic regions. Arrows indicate transcription directions.

expressed $^{48}$. As revealed by our real-time quantitative reverse transcription PCR (qRT-PCR) experiment and sRNA-seq, the expression level of MIR398a was extremely low (Supplementary Fig. 3 and Supplementary Table 2), while MIR398b and $M I R 398 c$ were very strongly expressed under the same experimental conditions.

To explore the temporal and spatial expression patterns of MIR398 and NAT398, the promoters of $M I R 398 \mathrm{a} / \mathrm{b} / \mathrm{c}$ and $N A T 398 b / c$ fused with the GUS gene were transferred separately into the Col ecotype of Arabidopsis. In $\mathrm{T}_{3}$ seedlings, pMIR398a:: GUS expression was examined in hypocotyls and leaf tips (Supplementary Fig. 3b). Both pMIR398b::GUS and pNAT398b:: GUS were constitutively expressed, with preferential expression observed in vascular tissues (Fig. 2a). pMIR398c::GUS expression was detected in all tissues, especially vascular tissues of seedlings (Fig. 2b), whereas pNAT398c::GUS expression was restricted to the vascular tissues of cotyledons and hypocotyls (Fig. 2b). These results indicate that pMIR398b::GUS and pNAT398b:: GUS were co-expressed in plant tissues, while pMIR398c::GUS and pNAT398c::GUS were partially co-expressed in cotyledons and hypocotyls. The similar expression patterns between
pMIR398::GUS and pNAT398::GUS suggest the possibility of shared regulatory mechanisms.

We separately overexpressed MIR398a, MIR398b, and MIR398c genes in $\mathrm{Col}$ under the control of the CaMV $35 \mathrm{~S}$ promoter. Overexpression of MIR398a was associated with higher levels of miR398 and lower levels of the miR398-targeted CSD1 gene compared with the wild-type (Fig. $2 \mathrm{~g}, \mathrm{~h}$ and Supplementary Table 3). In repeated trials, miR398 levels unexpectedly declined in transgenic lines of p35S::MIR398b and p35S::MIR398c, causing upregulation of the CSD1 gene (Figs. $2 \mathrm{c}-\mathrm{f}$ and $\mathrm{S} 4 \mathrm{a}-\mathrm{b}$ ). Because MIR398b and MIR398c primary transcript levels were higher in p35S::MIR398b and p35S::MIR398c lines compared with wild-type plants, they were obviously not silenced in these transgenic lines. We thus wondered whether the unexpected decline in miR398 levels in $p 35 S:: M I R 398 b$ and $p 35 S:: M I R 398 c$ transgenic lines was due to the efficiency of $p 35 S:: M I R 398 b$ and $p 35 S::$ MIR398c constructs, which may not have been able to properly produce miR398. We therefore transiently expressed p35S:: MIR398a, p35S::MIR398b, and p35S::MIR398c constructs in tobacco (Nicotiana benthamiana). In the transgenic tobacco plants, the accumulation of miR398a and miR398b/c was higher 


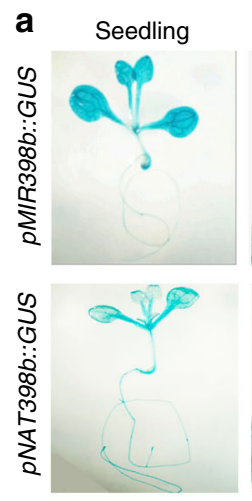

C

e

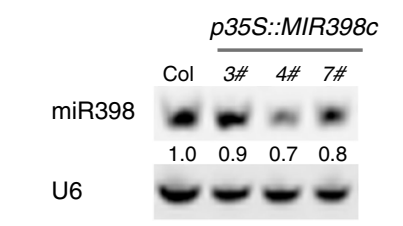

g

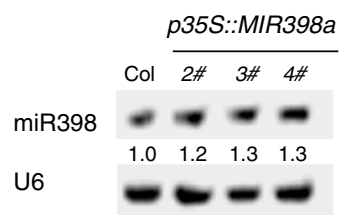

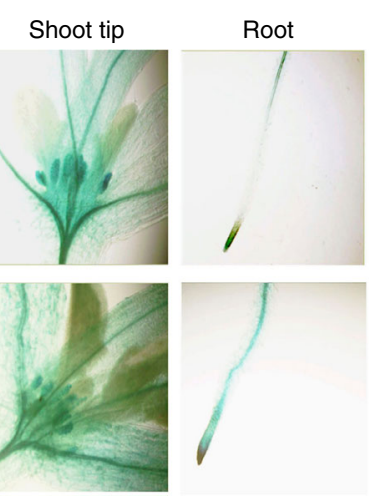
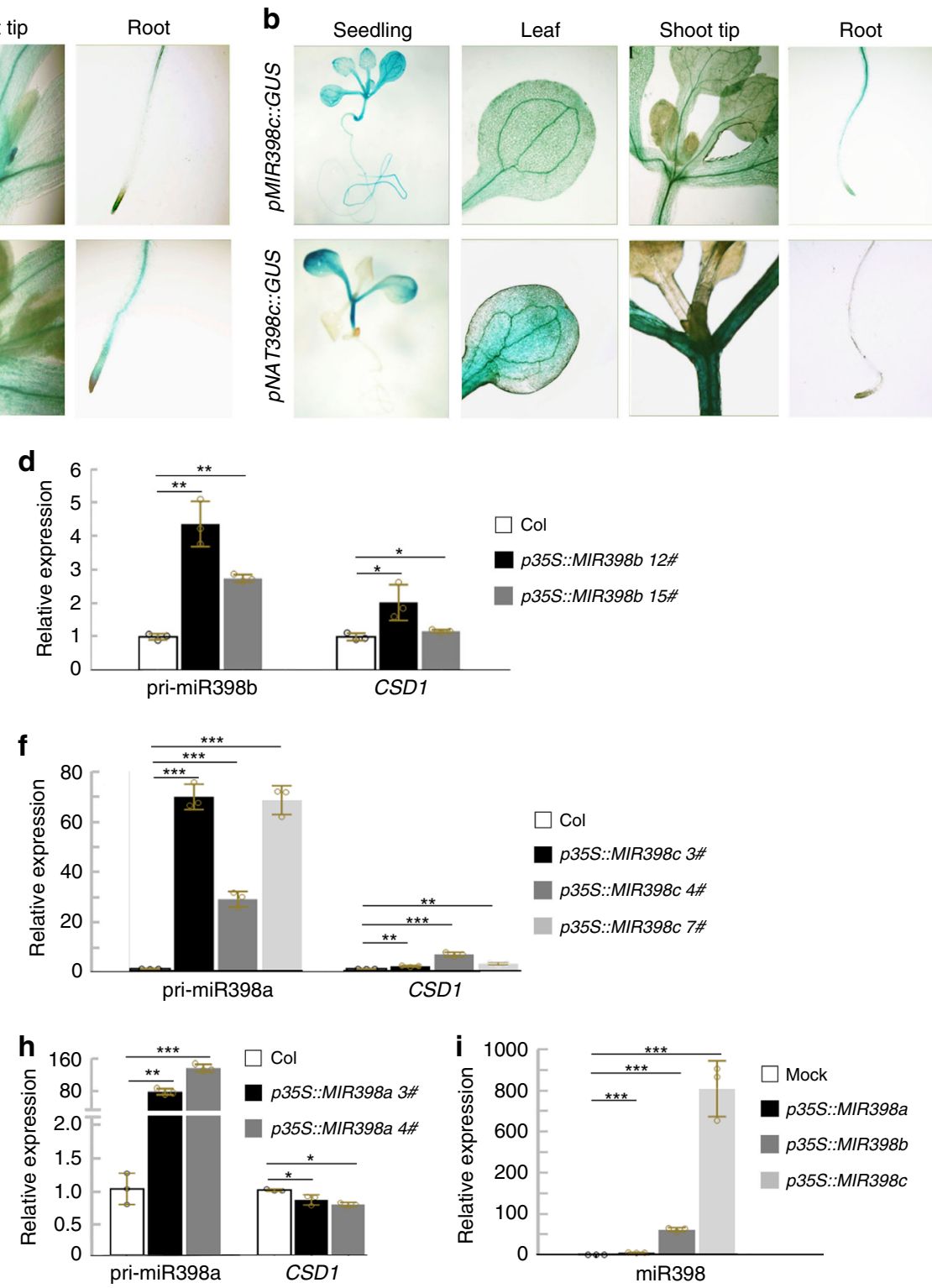

Fig. 2 Reduction in miR398 levels by overexpression of MIR398b and MIR398c genes. a, b GUS signals showing temporal and spatial expression of MIR398b and NAT398b (a) and MIR398c and NAT398c (b). The experiments were repeated at least three times with similar results, and data from one representative experiment are shown. c, e, $\mathbf{g}$ Northern blot showing miR398 accumulation in p35S::MIR398b (c), p35S::MIR398c (e), and p35S::MIR398a (g) seedlings. d, f, h Results of qRT-PCR showing relative expression levels of related genes in p35S::MIR398b (d), p35S::MIR398c (f), and p35S::MIR398a (h) seedlings. i miR398 accumulation in tobacco plants transiently expressing MIR398 genes. Error bars are the mean \pm SD. Significant differences were determined by one-tailed student's $t$-test $\left({ }^{\star} P<0.05 ;{ }^{\star \star} P<0.01 ;{ }^{\star \star \star} P<0.001\right)$.

than in control plants (Fig. 2i), thus indicating that all of the MIR398 overexpression constructs were functioning in plants. We therefore inferred that some genetic elements in A. thaliana may be involved in repressing miR398 processing in $p 35 S:$ : MIR398b and $p 35 S:: M I R 398 c$ plants but not in $p 35 S:: M I R 398 a$ plants.

Knock down of NAT398b/c upregulates MIR398b and MIR398c. To test whether NAT398b and NAT398c are involved in regulating the expression of MIR398b and MIR398c, we knocked down the expression levels of NAT398b and NAT398c by constructing artificial miRNAs of NAT398b and NAT398c. NAT398b overlaps with $M I R 398 b$ in pre-miR398b regions, while NAT398c overlaps with MIR398c elsewhere. To design artificial miRNAs, we selected appropriate fragments in upstream regions of $N A T 398 b$ and NAT398c. p35S::amiR-NAT398b, an artificial miRNA specific for
NAT398b in a MIR319a backbone ${ }^{12}$, yielded three transgenic lines when transferred into the Col ecotype. Expression levels of NAT398b declined in p35S::amiR-NAT398b plants concomitant with an increase in pri-miR398b (Fig. 3a). Decreased expression of NAT398c and increased levels of pri-miR398c were also observed in transgenic lines of $p 35 S:: a m i R-N A T 398 c$ plants (Fig. 3b). In addition, relative expression levels of CSD1 decreased in $p 35 S:: a m i R$ NAT398b and p35S::amiR-NAT398c plants (Fig. 3a-b). These results revealed that $N A T 398 b$ and NAT398c negatively regulate primiR398b and pri-miR398c levels.

To avoid the possibility of bias, we used the CRISPR/Cas9 approach to edit NAT398b. The resulting transgenic lines were designated as CRNAT398b plants. The proteins of the Core-2/Ibranching enzyme encoded by NAT398b were truncated because of premature translational termination, but this had no effect on the expression level of pri-miR398b (Figs. 3e and S5). This 
a
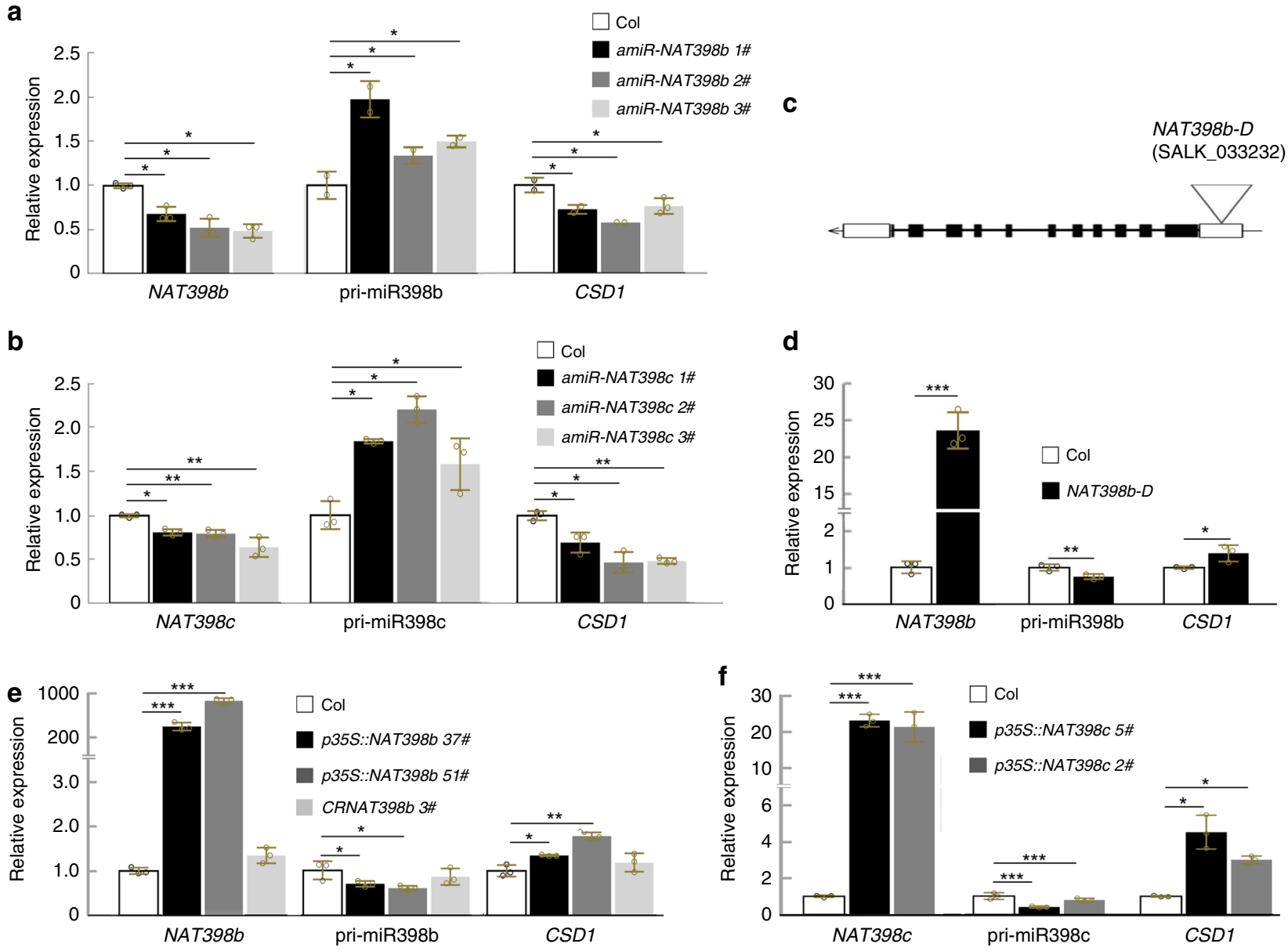

Fig. 3 Reduction in pri-miR398b/c levels by NAT398b/c. a, b Relative expression levels of related genes in p35S::amiR-NAT398b (a) and p35S::amiRNAT398c (b) seedlings. c Diagram showing the location of the T-DNA insertion in the NAT398b-D mutant. $\mathbf{d}$ Relative expression levels of related genes in NAT398b-D seedlings. e, f Relative expression levels of genes in p35S::NAT398b and CRNAT398b (e) and p35s::NAT398c (f) seedlings. Error bars are the mean \pm SD. Significant differences were determined by one-tailed student's $t$-test $\left({ }^{\star} P<0.05\right.$; $\left.{ }^{\star \star} P<0.01 ;{ }^{\star \star \star} P<0.001\right)$.

result excluded the possibility that pri-miR398b was affected by NAT398b proteins.

We crossed the $p 35 S:: M I R 398 b$ plants with $p 35 S:: a m i R-$ NAT398b or CRNAT398b plants to analyze the genetic interaction between MIR398b and NAT398b. Expression levels of pri-miR398b and miR398 in $p 35 S:: M I R 398 b \times p 35 S:: a m i R-$ NAT398b plants were significantly higher than those in $p 35 S::$ MIR398b plants (Supplementary Fig. 6), whereas levels in $p 35 S:$ : $M I R 398 b \times C R N T 398 b$ plants were not significantly higher than those in p35S::MIR398b plants (Supplementary Fig. 6). These results indicate that NAT398b transcripts, rather than NAT398b proteins, impaired the expression of pri-miR398b and miR398 in p35S::MIR398b plants.

Overexpressing NAT398b and NAT398c represses the processing of miR398. To test how NAT398b and NAT398c regulate pri-miR398b and pri-miR398c, we next overexpressed NAT398b and NAT398c in Col. In p35S::NAT398b plants, the upregulation of NAT398b was concomitant with downregulation of primiR398b (Fig. 3e), which caused a decline in miR398 accumulation (Supplementary Fig. 7a and Supplementary Table 3) and upregulated CSD1 expression (Figs. $3 \mathrm{e}$ and S4c); this indicates that NAT398b negatively regulates pri-miR398b. We additionally selected NAT398b-D, a dominant mutant of NAT398b (NAT398b-D, SALK_033232) (Fig. 3c). In NAT398b-D plants, the expression of NAT398b increased, while pri-miR398b declined and CSD1 was upregulated (Fig. 3d). Similarly, overexpression of NAT398c had negative effects on pri-miR398c (Figs. 3f, S4d, and S7b).

$N A T 398 b / c$ impair the stability and cleavage processing of pri-miR398b/c. To ascertain whether MIR398b and MIR398c genes are transcriptionally regulated by their cis-NATs, we investigated the occupancy of RNA polymerase II (Pol II) at the promoters of MIR398b and MIR398c in p35S::NAT398b and p35S::NAT398c plants, respectively. No significant difference was observed in Pol II occupancy between wild-type and transgenic plants (Supplementary Fig. 8). This result suggests that $N A T 398 b$ and $N A T 398 c$ do not regulate $M I R 398 b$ and $M I R 398 c$ genes at the transcriptional level.

The regulation of MIRNAs by their cis-NATs might also take place via the formation of an RNA duplex, which alters the secondary or tertiary structure of $\mathrm{RNA}^{23,49,50}$. To determine whether NAT398b/c and pri-miR398b/c form dsRNA, we devised an in vivo RNase protection assay based on the differential susceptibility of single-stranded RNAs (ssRNAs) and doublestranded RNAs (dsRNAs) to RNase $A+T^{51}$. RNase-treated or untreated Col RNA was used as a template for strand-specific qRT-PCR along with primers located in complementary or non-complementary regions. According to the results, noncomplementary regions of NAT398b/c and pri-miR398b/c were degraded by RNase $\mathrm{A}+\mathrm{T}$, while complementary regions were 
a

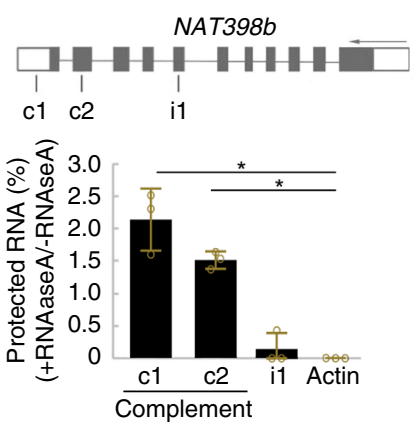

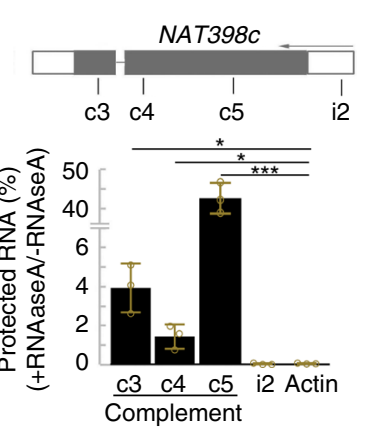

c
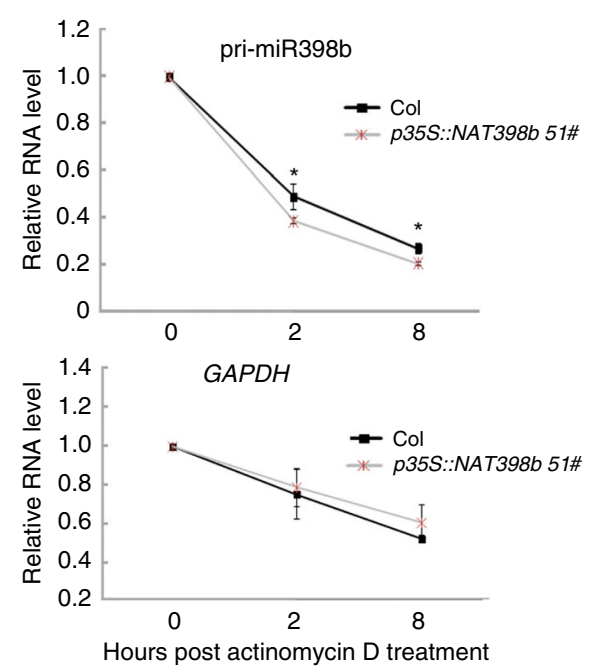

e

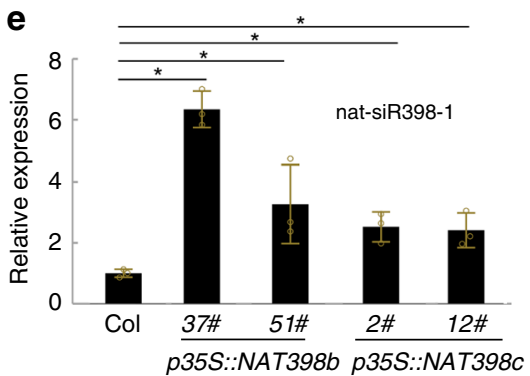

b

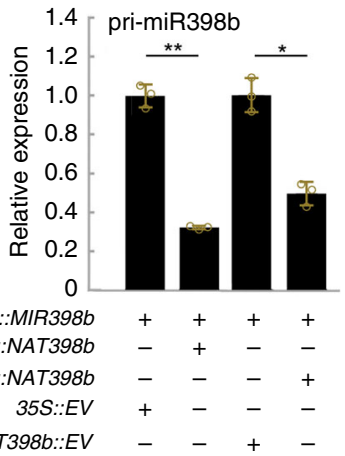

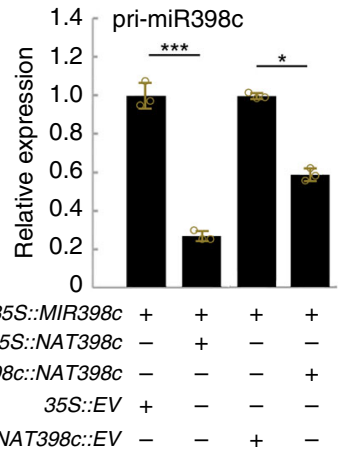

d
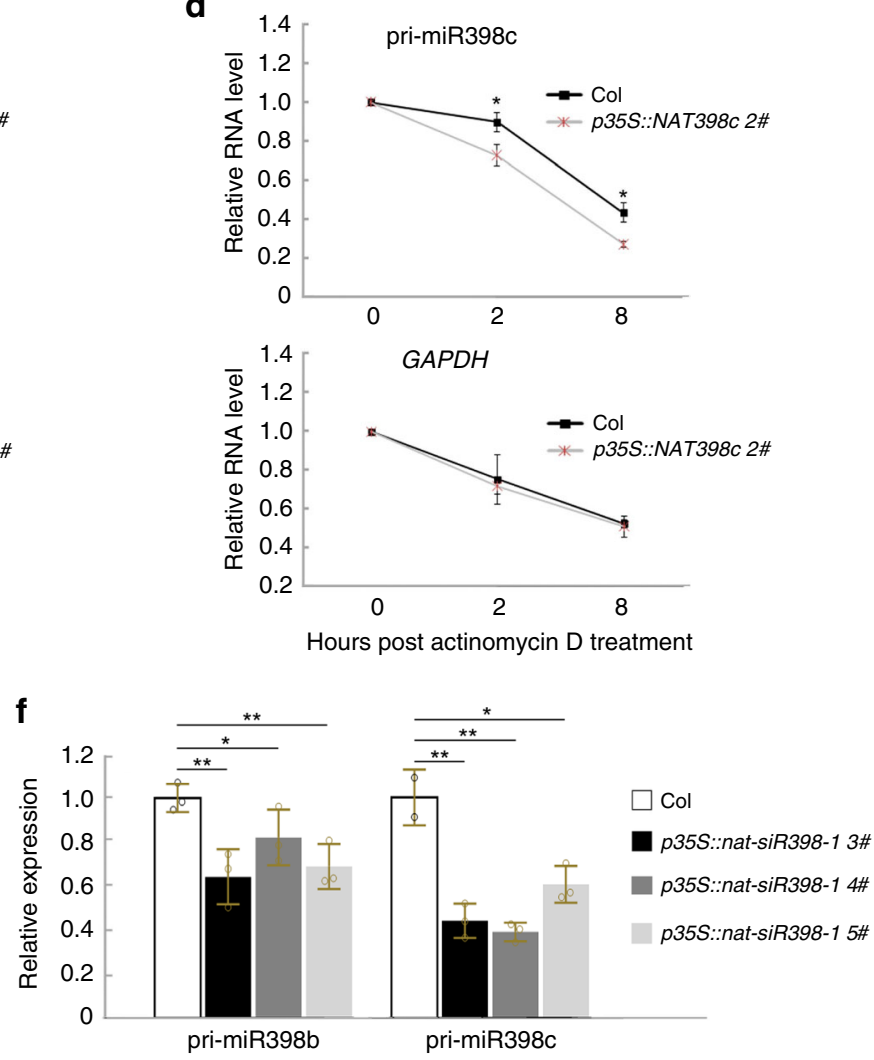

Fig. 4 Mechanisms of regulation of pri-miR398b/c by NAT398b/c. a RNase protection assays showing double-stranded RNA formation between NAT398b/c and pri-miR398b/c. Primers c1, c2, c3, c4, and c5 are located in complementary regions between pri-miRNAs and NAT RNAs; primers i1 and i2 are located in non-complementary regions. b Relative expression levels of MIR398b/c in tobacco plants transiently expressing NAT398b/c and MIR398b/c. c, d Relative RNA levels at different time points after treatment with actinomycin D (100 $\mu \mathrm{g} / \mathrm{mL})$ in Col and p35S::NAT398b (c) and p35S:: NAT398c (d) lines. e nat-siR398b-1 accumulation in p35S::NAT398b and p35S::NAT398c seedlings. f Relative expression levels of MIR398b and MIR398c in p35S::nat-siR398b-1 seedlings. Error bars are the mean \pm SD. Significant differences were determined by one-tailed student's $t$-test $\left({ }^{\star} P<0.05\right.$; ${ }^{\star \star} P<0.01$; $\left.{ }^{\star \star \star} P<0.001\right)$.

protected from degradation (Fig. 4a). Similar degradation was observed in the case of transcripts of ACTIN2, used as a negative control. These results suggest that pri-miR398b/c and their cisNATs form double-stranded RNAs in vivo.

Because RNA duplexes have been reported to influence RNA stability $23,49,50$, we examined whether NAT398b/c regulate primiR398b/c RNA stability. We first transiently expressed $p 35 S::$ MIR398b synchronously with p35S::NAT398b or pNAT398b:: $N A T 398 b$ in tobacco and found that the expression level of MIR398b was downregulated compared with plants expressing p35S::MIR398b or empty vectors (Fig. 4b). We then co-expressed p35S::MIR398b and p35S::NAT398b in Col and found that the expression level of MIR398b was also downregulated relative to plants only expressing p35S::MIR398b (Supplementary Fig. 9). A similar inhibitory effect on MIR398c due to NAT398c was observed when we co-expressed $p 35 S:: M I R 398 c$ and either $p 35 S::$ NAT398c or pNAT398c::NAT398c in tobacco and Arabidopsis (Figs. $4 \mathrm{~b}$ and S9). These results indicate that NAT398b and NAT398c impair the stability of pri-miR398b and pri-miR398c transcripts, respectively.

We further measured the RNA decay rate of pri-miR398b/c in Col, p35S::NAT398b and p35S::NAT398c lines via treatment with the transcriptional inhibitor actinomycin $\mathrm{D}$. We found that the decay rates of pri-miR398b and pri-miR398c were higher in $p 35 S:: N A T 398 b$ and $p 35 S:: N A T 398 c$ plants than in Col (Figs. $4 c, d$ ). In contrast, the decay rates of GAPDH transcripts in 
Col, p35S::NAT398b and p35S::NAT398c lines were indistinguishable (Fig. 4c, d). These results confirm that NAT398b and NAT398c impair the stability of pri-miR398b and pri-miR398c transcripts, respectively.

Aberrance of cleavage sites in pri-miRNAs causes alterations in pre-miRNA sequences, which likely leads to the production of inaccurate miRNAs. To understand whether NAT398 affects primiR398 cleavage, we collected RNA samples of 3-week-old seedlings and carried out a $5^{\prime}$ RACE-PCR analysis to detect the cleavage sites in $5^{\prime}$ single-stranded RNA regions of pri-miR398b. The percentage of inaccurate cleavage sites in p35S::amiR-NAT398b plants was lower than in the wild-type, whereas the percentage was much higher in p35S::NAT398b lines (Supplementary Table 4). Small RNA (20-70 nt) paired-end sequencing further revealed a subset of degradation products from pri-miR398b, and the accumulation of these fragments was also much higher in $p 35 S:$ : $N A T 398 b$ plants than in the wild-type (Supplementary Table 5). These results suggest that NAT398b overexpression increases the number of incorrect pri-miR398b cleavage sites and thus interferes with its accurate processing.

The $3^{\prime}$-UTR region of NAT398b is complementary to primiR398b, but its coding sequence is not. To examine whether the $N A T 398 b$ 3'-UTR region affects the expression of $M I R 398 b$, we overexpressed NAT398b cDNA and NAT398b coding sequences in Col. In this way, we generated a version of $N A T 398 b$ that maintains its ORF but loses $315 \mathrm{nt}$ sequence complementarity to pri-miR398b. In p35S::NAT398b(cDNA) plants, NAT398b was upregulated, concomitant with downregulation of pri-miR398b (Supplementary Fig. 10). In contrast, the upregulation of NAT398b in p35S::NAT398b(CDS) plants did not accompany downregulation of pri-miR398b (Supplementary Fig. 10). This result suggests that the NAT398b $3^{\prime}$-UTR region complementary to pri-miR398b is crucial for pri-miR398b expression.

Overexpression of nat-siR398 reduces pri-miR398b and primiR398c levels. The regulation of $M I R 398 b / c$ by their cis-NATs may be also achieved via the formation of cis-NAT-derived small interfering RNAs (nat-siRNAs), which may degrade pri$\mathrm{miR} 398 \mathrm{~b} / \mathrm{c}$ through siRNA-induced gene silencing. sRNA deep sequencing revealed several types of nat-siR398 entities-the siRNAs derived from NAT398 transcripts. Among them, natsiR398b-1 and nat-siR398b-2 are located in the region of NAT398b and MIR398b overlap (Fig. 1b). Both nat-siRNAs were accumulated at much higher levels in p35S::NAT398b plants than in the wild-type (Figs. $4 \mathrm{e}$ and S11b).

To define genetic components required for the formation of nat-siRNAs, the expression level of nat-siR398b-1 was examined in some small RNA biogenesis mutants. We found that $d c l 2 / 3 / 4$ triple mutations or the $r d r 6$ mutation blocked nat-siR398b-1 accumulation, while $d c l 1, d c l 2, d c l 3, d c l 4$, and $r d r 2$ single mutations had little effect on nat-siR398b-1 accumulation (Supplementary Fig. 12). These results suggest that RDR6 is required for nat-siR398b-1 accumulation and that DCL2, DCL3 and DCL4 play redundant roles in nat-siR398b-1 production.

To determine the function of nat-siR398b-1, we constructed an artificial miRNA vector with a MIR319a backbone ${ }^{12}$ and overexpressed nat-siR398b-1 in Col under the control of the CaMV 35S promoter. Expression levels of both pri-miR398b and pri-miR398c declined in the p35S::nat-siR398b-1 plants (Fig. 4f). This fact suggests that nat-siR398-1 reduces pri-miR398b and primiR398c levels.

Expression of NAT398 is upregulated by overexpression of MIR398 genes. To examine whether the expression of MIR398b/c genes affects the expression of their antisense genes, we investigated
NAT398b/c expression in p35S::MIR398b/c plants. According to our data, expression levels of NAT398b and NAT398c were much higher in p35S::MIR398b and p35S::MIR398c plants than in the wild-type (Fig. 5a, b). We also discovered that expression levels of NAT398b and NAT398c were reduced in p35S::nat-siR398-1 plants (Fig. 5c). These results indicate that MIR398b and MIR398c genes positively regulate their antisense genes.

SALK_038698 mutants harboring a T-DNA insertion in the promoter region of $M I R 398 \mathrm{c}$ were applied to confirm the above finding (Fig. 5d). The SALK_038698 mutant displayed lower MIR398c expression and was designated as mir398c (Figs. 5e and S7c). The expression of NAT398c was downregulated in the mir398c mutant (Fig. 5e). These results confirm that the expression of MIR398b and MIR398c genes positively regulates their respective cis-NATs.

We next examined how MIR398b and MIR398c regulate their cis-NATs. We found that MIR398b and MIR398c overexpression enhanced the transcriptional activity of NAT398b and NAT398c, respectively (Fig. 5a, b). ChIP-qPCR analysis also revealed that recruitment of Pol II at the promoter of $N A T 398 b$ or NAT398c was respectively increased in $p 35 S:: M I R 398 b$ and p35S::MIR398c plants (Fig. 5g). These results suggest that MIR398b and MIR398c directly activate the transcription of their cis-NATs.

To reveal differences in the RNA interference of NATs between pri-miR398 and mature miR398, we constructed an artificial $\mathrm{miR} 398 \mathrm{~b} / \mathrm{c}(\mathrm{amiR} 398 \mathrm{~b} / \mathrm{c})$ vector with a MIR319a backbone $^{12}$. In p35S::amiR398b/c seedlings, CSD1 and CSD2 expressions were notably downregulated compared with the wild-type (Fig. 5f), whereas NAT398b and NAT398c were unchanged (Fig. 5f). This fact implies that mature miR398 can silence its target genes instead of its cis-NATs.

NAT398b/c attenuate plant thermotolerance. Previous researchers have reported that cis-NATs regulate plant temperature response $\mathrm{e}^{52,53}$. In addition, pri-miR398b, pri-miR398c and mature miR398 are induced by heat stress in Arabidopsis ${ }^{42}$. To examine whether the cis-NATs of MIR398b/c are heat-responsive, we subjected Col seedlings to a heat treatment of $38^{\circ} \mathrm{C}$ for $1 \mathrm{~h}$. Northern blotting revealed that miR398 accumulation in seedlings was much higher at $38^{\circ} \mathrm{C}$ than at $22^{\circ} \mathrm{C}$ (Fig. 6a). Real-time PCR indicated that pri-miR398b and pri-miR398c expressions were induced by heat stress, while NAT398b and NAT398c expressions were inhibited (Fig. 6b). At the same time, the expression of the miR398-targeted CSD1 gene was downregulated under heat treatment (Supplementary Fig. 13c). Under heat treatment, expression levels of pri-miR398b and miR398 were lower and those of CSD1 were higher in $p 35 S:: N A T 398 b$ plants than in the wild-type (Supplementary Fig. 13a-c), thus indicating that NAT398b affects heat responses of miR398 and CSD1.

To gain insight into the mechanism of MIR398/NAT398 regulation under heat stress, $M I R 398 b / c^{-}$and $N A T 398 b / c$ promoter-GUS transgenic plants were subjected to the same heat stress conditions and analyzed for GUS activity. Analysis of the seedlings revealed an increase in GUS signal intensity after stress treatment in pMIR398b/c::GUS but a decrease in pNAT398b/c:: GUS (Supplementary Fig. 13d). These results indicate that heat stress activates the transcription of $M I R 398 b / c$ genes and suppresses the transcription of $N A T 398 \mathrm{~b} / \mathrm{c}$ genes.

The redox status of plants is reported to be influenced by heat stress, which affects expression of heat-responsive genes ${ }^{42,54-56}$. We thus measured $\mathrm{H}_{2} \mathrm{O}_{2}$ levels in mutant and transgenic plants. Similar to the findings of Guan et al. (2013), csd1 plants accumulated much higher levels of $\mathrm{H}_{2} \mathrm{O}_{2}$ (as indicated by 3,3'-diaminobenzidine staining) under heat stress (Fig. 6c), while p35S::CSD1 plants accumulated much lower $\mathrm{H}_{2} \mathrm{O}_{2}$ levels (Supplementary Fig. 15). 

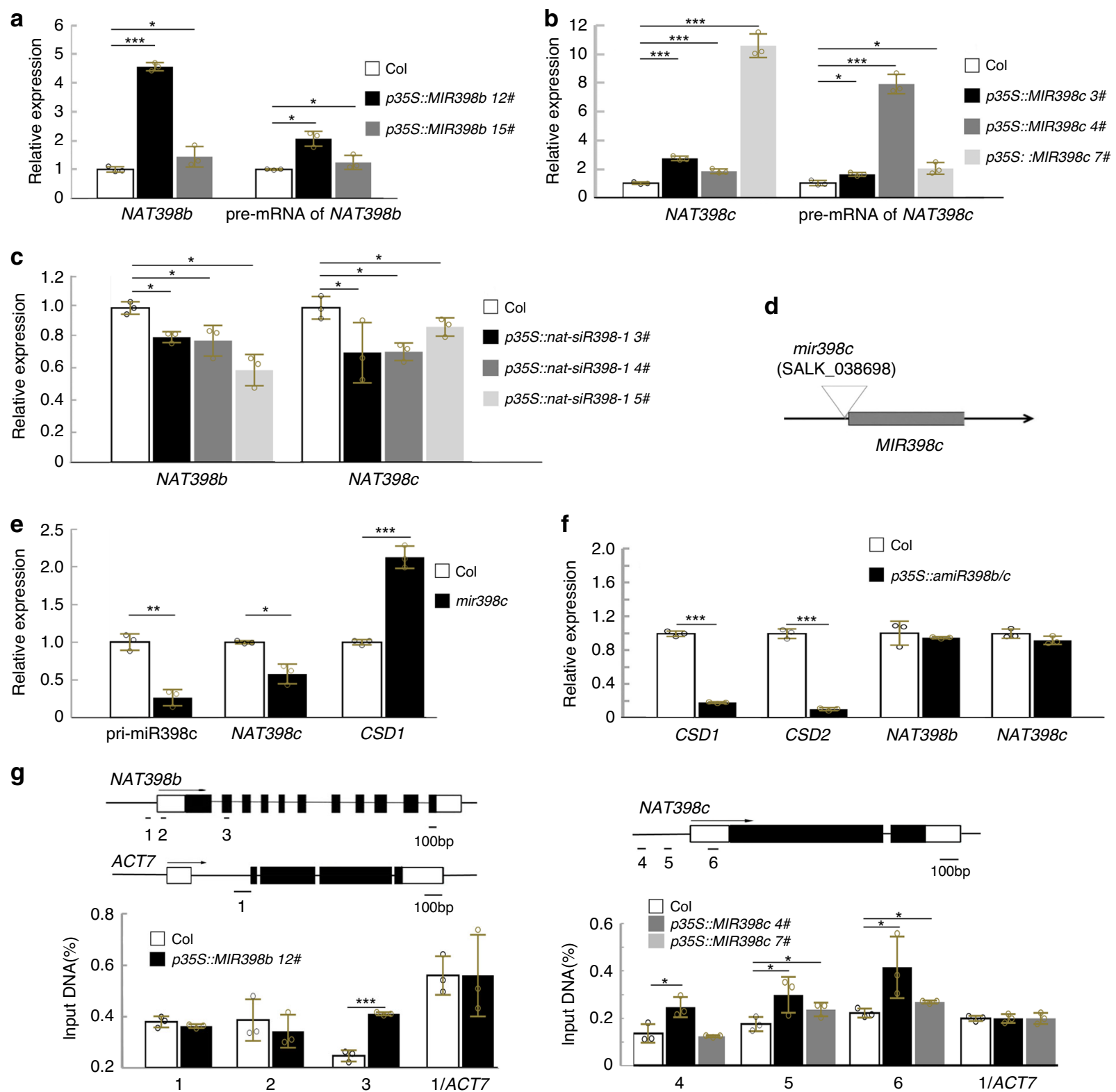

Fig. 5 MIR398b and MIR398c activation of their cis-NATs. a, b Relative expression levels of genes in p35S::MIR398b (a) and p35S::MIR398c (b) seedlings. c Relative expression levels of NAT398b and NAT398c in p35S::nat-siR398b-1 seedlings. $\mathbf{d}$ Diagram showing the location of the T-DNA insertion in the mir398c mutant. e, $\mathbf{f}$ Results of qRT-PCR showing gene relative expression levels in mir398c mutant (e) and p35S::amiR398b/c (f) seedlings. $\mathbf{g}$ ChIP analysis showing relative accumulation of RNA Pol II in p35S::MIR398b and p35S:::MIR398c plants. Error bars are the mean \pm SD. Significant differences were determined by one-tailed student's $t$-test $\left({ }^{\star} P<0.05 ;{ }^{\star \star} P<0.01\right.$; $\left.{ }^{\star \star \star} P<0.001\right)$.

Compared with the wild-type, $p 35 S:: M I R 398 a$ plants accumulated slightly higher levels of $\mathrm{H}_{2} \mathrm{O}_{2}$ under control conditions and much higher levels under heat stress (Supplementary Fig. 15). In contrast, $\mathrm{H}_{2} \mathrm{O}_{2}$ accumulation was lower in p35S::MIR398b and p35S:: MIR398c plants under both control and heat stress conditions compared with the wild-type (Supplementary Fig. 15). These results indicate that altered expression of miR398 changed the redox status of plants.

We also investigated whether $N A T 398 b / c$ could alter the redox status of transgenic plants under heat stress. Compared with wildtype plants, p35S::amiR-NAT398b/c plants accumulated slightly higher levels of $\mathrm{H}_{2} \mathrm{O}_{2}$ under control conditions (Fig. 6c) and much higher levels under heat stress (Fig. $6 \mathrm{c}$ ). In contrast, $p 35 \mathrm{~S}::$ NAT398b and p35S::NAT398c plants accumulated slightly lower levels of $\mathrm{H}_{2} \mathrm{O}_{2}$ under control conditions compared with wild-type plants and much lower levels under heat stress (Fig. 6c). $\mathrm{H}_{2} \mathrm{O}_{2}$ accumulation in CRNAT398b 3\# mutants was similar to wild-type plants under both control and heat stress conditions (Fig. 6c). Taken together, these results demonstrate that NAT398b and NAT398c alter the redox status of plants by regulating miR398 accumulation.

Next, we examined the thermotolerance of mutants and transgenic plants. We found that $c s d 1$ mutants were insensitive to heat stress, as indicated by increased survival rates of flowers and increased percentages of green leaves under heat stress (Fig. 6d-f). In contrast, transgenic plants overexpressing CSD1 gene displayed increased sensitivity to heat shock (Supplementary Fig. 16). We also measured the thermotolerance of p35S:: MIR398a, p35S::MIR398b and $p 35 S:: M I R 398 c$ plants. We found that $p 35 S:: M I R 398 a$ plants had stronger heat resistance, 
a

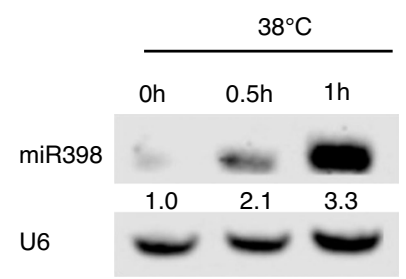

b

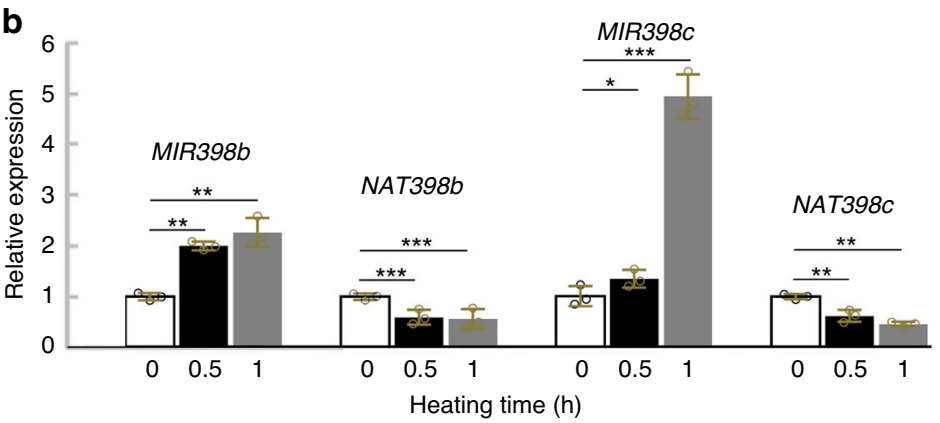

C
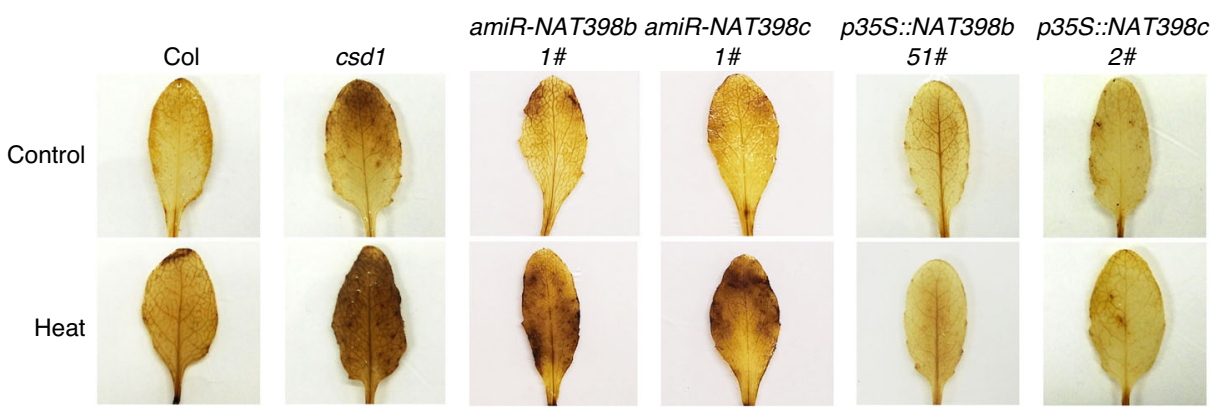

CRNAT398b

d

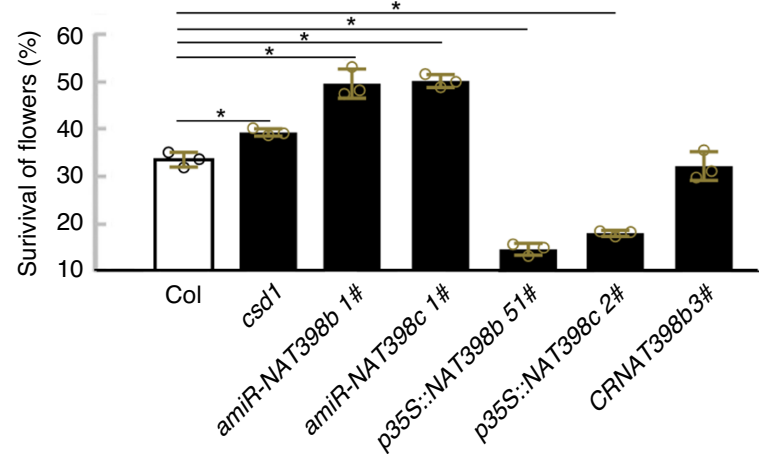

f

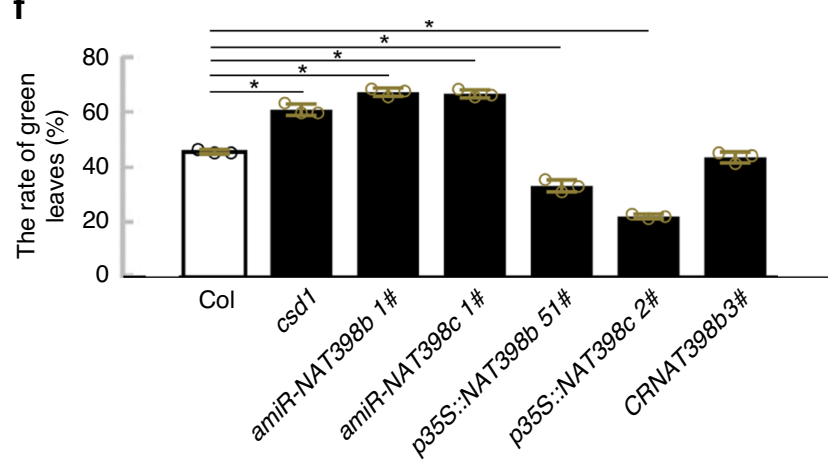

$\mathbf{e}$

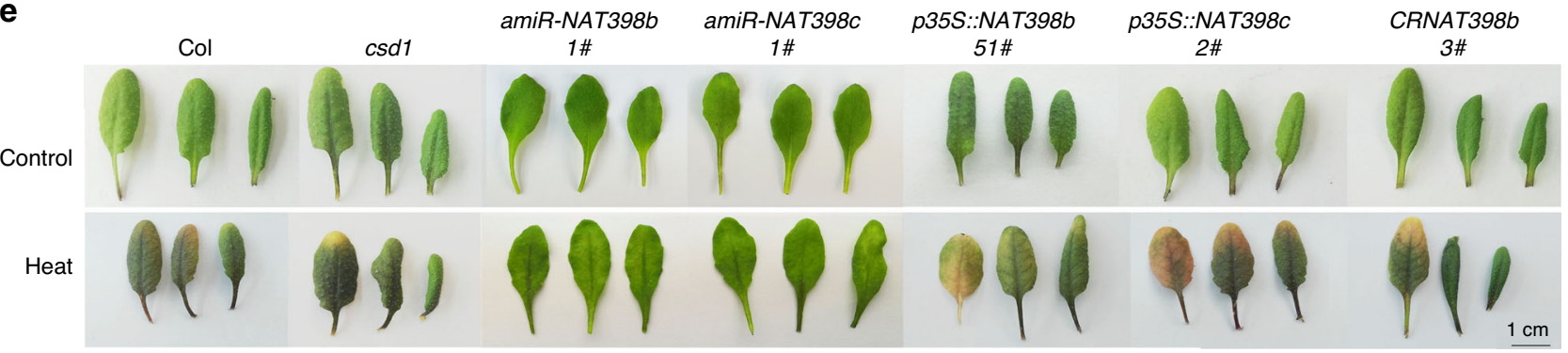

Fig. $6 \mathbf{N A T 3 9 8 b / c}$ attenuation of $\mathbf{H}_{\mathbf{2}} \mathbf{O}_{\mathbf{2}}$ accumulation and thermotolerance in Arabidopsis. a Northern blot showing miR398 accumulation in seedlings exposed to high temperature for 0 to $1 \mathrm{~h}$. $\mathbf{b}$ Relative expression levels of MIR398b, NAT398b, MIR398c, and NAT398c in seedlings exposed to high temperature for $0-1 \mathrm{~h}$. Error bars are the mean $\pm \mathrm{SD}$ ( $n=3$ biological replicates). c DAB staining for $\mathrm{H}_{2} \mathrm{O}_{2}$ levels in transgenic and mutant plants. $\mathbf{d}$ Survival rate of flowers of wild-type, csd1, p35S:::amiR-NAT398b, p35S::amiR-NAT398c, p35S::NAT398b, p35s::NAT398c, and CRNAT398b plants under heat stress. e, f Phenotypes of detached leaves $(\mathbf{e})$ and the rate of green leaves (f) of wild-type, csd1, p35S:::amiR-NAT398b, p35S:::amiR-NAT398c, p35S::NAT398b, p35S::NAT398c, and CRNAT398b plants. Error bars are the mean \pm SD $(n=3$ biological replicates, a replicate constitutes 60 biologically independent samples for $\mathbf{d}$, and 12 biologically independent samples for $\mathbf{f}$ ). Significant differences were determined by one-tailed student's $t$-test $\left({ }^{\star} P<0.05 ;{ }^{\star \star} P<0.01\right.$; $\left.{ }^{\star \star \star} P<0.001\right)$.

whereas p35S::MIR398b and p35S::MIR398c plants were heat sensitive, as expected (Supplementary Fig. 16). These results indicate that the miR398-CSD1 regulon modulates plant thermotolerance.

We additionally measured the thermotolerance of $p 35 S:: a m i R-$ NAT398b, p35S::amiR-NAT398c, p35S::NAT398b, p35S::NAT398c, and CRNAT398b plants. According to our measurements, $p 35 S:$ : $a m i R-N A T 398 b$ and p35S::amiR-NAT398c plants were more tolerant to heat stress compared with the wild-type, while p35S::NAT398b and p35S::NAT398c plants were more sensitive (Fig. 6d-f). CRNAT398b plants, however, exhibited no obvious differences compared with wild-type plants (Fig. 6d-f). These results suggest that $N A T 398 b / c$ attenuate plant thermotolerance by regulating pri-miR398 processing and the expression of miR398 target genes, thus indicating the important role of $\mathrm{NAT} 398 \mathrm{~b} / \mathrm{c}$ in the fine-tuning of plant thermotolerance. 


\section{Discussion}

Gene expression regulated by miRNAs and cis-NATs is well known in eukaryotes ${ }^{1,57}$, but the molecular relationship between MIRNA genes and their cis-NATs has rarely been documented ${ }^{38}$. In the present study, MIR398 genes in both $B$. rapa and $A$. thaliana were found to have cis-NATs. Sense-antisense regulatory loops between coding genes and long noncoding genes are usually widely present ${ }^{58}$. Here, we uncovered the existence of regulatory loops between coding sequences and pri-miRNAs.

In our previous study, we identified eight cis-NATs responsive to high temperature in $B$. rapa that correspond to precursors of miRNAs ${ }^{16}$. Using a combination of RNA-seq data and mRNA sequences, we identified 22 cis-NATs complementary to MIRNA genes in Brassica rapa and 25 MIRNA cis-NATs in Arabidopsis. We further found that MIR398 genes and their cis-NATs are conserved in Brassicaceae species, which indicates that cis-NATs of MIRNA are widely present in plants. Thousands of NATs have been identified in plants and implicated in seedling light response $\mathrm{s}^{59}$, flowering time control ${ }^{60}$, reproduction ${ }^{61}$, and biotic and abiotic stress responses $31,33,34,53,62,63$, but only a handful have been experimentally characterized. We expect that an increasing number of cis-NATs of MIRNA will be discovered under various conditions.

MiRNAs are derived from noncoding MIRNA genes and control plant growth and physiology through silencing of their target genes ${ }^{64,65}$. For genetic manipulation of agricultural traits, MIRNA genes have been broadly utilized ${ }^{10}$. Unfortunately, many MIRNA vectors do not work in transgenic plants. For example, overexpression of the MIR398b gene in Arabidopsis fails to elevate miR398 accumulation ${ }^{13}$. For identification of MIRNA gene functions in plants, artificial miRNAs are designed according to changes in RNA secondary or tertiary structures ${ }^{12}$. How these MIRNA genes lose their miRNA biogenesis ability remains unclear. Long noncoding RNAs (lncRNAs) have been reported to inhibit the expression of mature-Sly-miR482a in Solanum lycopersicum $^{39}$. In the present study, we found that miRNA processing of MIR398 family genes is disrupted by their cis-NATs transcribed from coding genes.

Changes in the secondary or tertiary structure of RNA alter the stability of noncoding RNAs ${ }^{23,49,50}$. The suppression of $N A T 398 b / c$ in the miRNA processing of MIR398b/c should be relevant to RNA secondary or tertiary structural deformation in the case of the existence of their NATs. These NATs may interrupt miRNA processing and undo the degradation or translational inhibition of their targets, in turn eventually influencing the key developmental processes of embryo, meristem, leaf and floral patterning 64,65 . Plants may exploit the balance between primiRNAs and their cis-NATs to facilitate plant development and stress response; any change in expression levels of cis-NATs can thus lead to an imbalance in biological responses. Understanding the complexity of the molecular relationship between primiRNAs and their cis-NATs is important. The existence and action of cis-NATs must be considered when MIRNA genes and artificial miRNAs are applied for the silencing of target genes in genetic transformation.

The expression of noncoding RNAs that transcribe in the opposite direction of protein-coding genes is often positively or negatively correlated with their cognate sense genes ${ }^{31,52,53}$. The cold-induced antisense transcript COOLAIR represses FLOWERING LOCUS C (FLC) transcription with increased H3K27me3 and decreased H3K36me3 levels in response to cold temperatures ${ }^{52,66}$. And the cold-induced antisense transcript SVALKA-asCBF1 suppresses $C B F 1$ by RNAPII collision stemming ${ }^{53}$. The transcriptional activating mechanisms adopted by $\operatorname{lncRNAs}$ are varied. Previous research has shown that $M A S$, a NAT-lncRNA produced from MADS AFFECTING FLOWERING4 (MAF4), recruits WDR5a and then guides COMPASS-like complexes to MAF4 to enhance histone-3 lysine-4 trimethylation (H3K4me3) ${ }^{31}$. Like MAS, HOTTIP and NeST RNAs bind the adaptor protein WDR5 and recruit the MLL complex to maintain $\mathrm{H} 3 \mathrm{~K} 4 \mathrm{me} 3$ and activate sense genes ${ }^{67,68}$. LRK antisense intergenic RNA binds histone modification proteins OsMOF and OsWDR5 to enhance H3K4me3 and $\mathrm{H} 4 \mathrm{~K} 16 \mathrm{ac}$ in the LRK1 gene region ${ }^{69}$. In addition, EVX1as increases the transcription of $E V X 1$ by facilitating the binding of the Mediator complex to the EVX1 region, leading to an active chromatin state $^{70}$. In this study, we found that noncoding genes MIR398b and MIR398c directly activate the transcription of their cis-NATs. Whether MIR398b and MIR398c regulate their cognate antisense genes through recruitment of COMPASS-like complexes or other mechanisms remains to be investigated.

In a previous investigation, overexpression of the MIR398b precursor sequence did not yield transgenic plants overexpressing miR398b, and the only recovered plants were those in which cosuppression had occurred ${ }^{13}$. In the present study, we overexpressed the pri-miR398b sequence and were likewise unable to obtain transgenic plants overexpressing miR398. Using the same primers to detect pri-miR398b levels, we found that pri-miR398b abundance was higher in our transgenic lines than in wild-type plants. This difference may be due to the overexpressed MIR398b sequence.

Heat stress is one of the major environmental stresses limiting plant growth, development and productivity; thus, plants have evolved special adaptive mechanisms to cope with high-temperature stress $^{71-74}$. A previous study has shown that HSFA1b and HSFA7b bind directly to the promoter regions of MIR398b to activate the transcription of $M I R 398 b^{42}$. In the present study, we found that heat stress activates the transcription of $M I R 398 b / c$ genes and suppresses the transcription of NAT398b/c genes. Whether heat stress regulates $N A T 398 \mathrm{~b} / \mathrm{c}$ genes by recruiting heat stress transcription factors or via other mechanisms remains to be investigated. Our findings provide another example of a regulatory mechanism for plant thermotolerance, namely, through regulation of cis-NATs of MIR398. In particular, the upregulation of miR398 by heat stress is caused by stress-induced activation of $M I R 398 \mathrm{~b} / \mathrm{c}$ transcription and the downregulation of NAT398b/c.

miR398 is an important conserved miRNA that is proposed to be linked to various abiotic and biotic stresses ${ }^{13,34,42-44}$. Although this study has revealed the important role of $N A T 398 \mathrm{~b} / \mathrm{c}$ in regulating plant thermotolerance, the roles of $N A T 398 \mathrm{~b} / \mathrm{c}$ in regulating other abiotic and biotic stresses need to be investigated.

$N A T 398 b$ is predicted to encode an acetylglucosaminyltransferase which suggests that NAT398b proteins have other biotic functions. NAT398c has additionally been reported to encode high-affinity nitrate transporter 2.7 (NRT2.7) ${ }^{47}$, and NAT398c proteins play a specific role in nitrate content and proanthocyanidin accumulation in seeds ${ }^{75,76}$ - which suggests that NAT398c proteins have other biotic functions. All of these results indicate that NAT398b and NAT398c are dual-functional. The regulatory relationship between MIR398 genes and their cisNATs is thus potentially useful to manipulate plant abiotic and biotic stresses tolerance. This fact adds an additional layer of complexity to the gene regulation of biological processes.

On the basis of our genetic and expression analyses, we propose a model for the regulatory loop between MIR398 genes and their cis-NATs (Fig. 7). In this model, MIR398b/c genes and $N A T 398 b / c$ are transcribed from opposing DNA strands at the same genomic locus, and MIR398b/c genes activate the transcription of their cis-NATs. NAT398b/c inhibit pri-miR398 processing by impairing the stability and accurate cleavage of pri$\mathrm{miR} 398 \mathrm{~b} / \mathrm{c}$, thus causing abnormal miR398 processing and biological responses controlled by the miRNA-targeted genes.

Overexpression of MIRNA genes to silence miRNA-targeted genes is generally applied to enhance the yield and quality of 


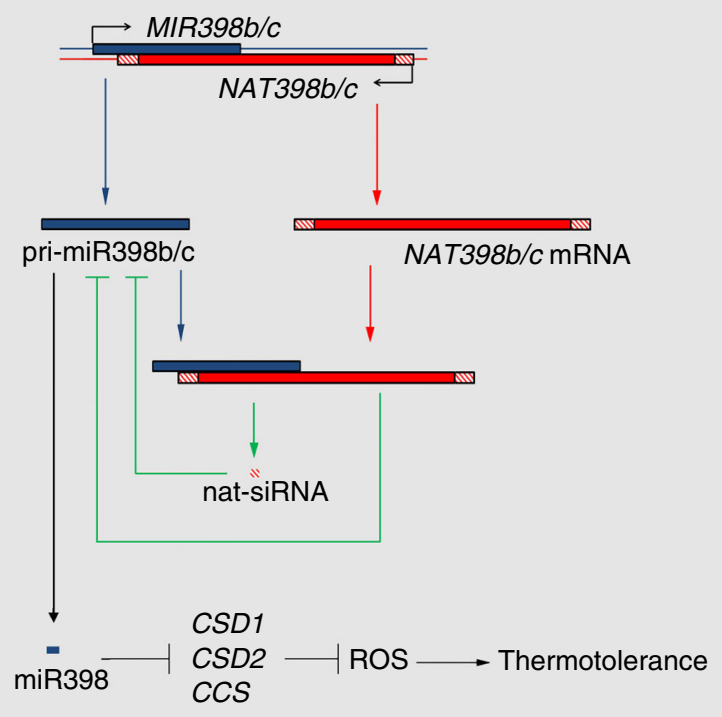

Fig. 7 Proposed model for the regulatory loop between MIR398b/c genes and their cis-NATs. MIR398b/c genes and their natural antisense transcripts NAT398b/c are transcribed from opposing DNA strands at the same genomic locus. NAT398b/c inhibit miR398 biogenesis and plant thermotolerance since they impair the stability and accurate cleavage of pri-miR398b/c.

agricultural crops ${ }^{10}$. The ability of MIRNA genes to suppress the processing of their miRNAs in the presence of their cis-NATs provides a reasonable explanation for the absence of accumulation of some miRNAs in transgenic plants overexpressing MIRNA genes. In addition, the effects of cis-NATs need to be avoided, or artificial miRNAs must be generated to silence their target genes. Because MIR398/NAT398 genes are conserved in Brassicaceae, the optimized relationship between MIR398 genes and their cis-NATs in Arabidopsis is potentially important for genetic manipulation of biological processes in various crops.

\section{Methods}

Plant materials and growth conditions. Arabidopsis thaliana ecotype Columbia (Col), used as the wild-type, served as the genetic background for transgenic plants. SALK_033232 (NAT398b-D) and SALK_038698 (mir398c) were obtained from the ABRC stock center (http://signal.salk.edu/cgi-bin/tdnaexpress). Seeds were surfacesterilized and sown on plates containing Murashige and Skoog (MS) medium supplemented with $1 \%$ sugar. After $2 \mathrm{~d}$ at $4{ }^{\circ} \mathrm{C}$, the plates were transferred to a phytotron under growth conditions of $22^{\circ} \mathrm{C}$ and $16 / 8$-h light/dark. For detection of heat response, $15-\mathrm{d}$-old seedlings were incubated at $38^{\circ} \mathrm{C}$, and plants grown at $22{ }^{\circ} \mathrm{C}$ served as controls.

Plant sRNA sequencing. For sRNA sequencing, 15-d-old seedlings of $A$. thaliana in MS medium were collected with two biological replicates. RNA samples were extracted using Trizol reagent (Invitrogen, Carlsbad, CA, USA) and treated with DNase I (Takara, Dalian, China) to remove DNA contamination. RNA samples prepared from seedlings were sent to WuXi AppTec (Shanghai, China) for sRNA sequencing.

\section{Gene cloning and generation of transgenic plants. MIR398a, MIR398b,}

MIR398c, NAT398b, and NAT398c genes were amplified from Col genomic DNA, and the PCR fragments were subcloned into pCAMBIA1301 binary vectors along with a CaMV $35 \mathrm{~S}$ promoter and eGFP. Fragments corresponding to the promoters of MIR398a, MIR398b, MIR398c, NAT398b and NAT398c were inserted into pCAMBIA1301 binary vectors upstream of $\beta$-glucuronidase (GUS) to replace the CaMV $35 \mathrm{~S}$ promoter. The destination vectors were introduced into Agrobacterium tumefaciens strain GV3101 (pMP90RK) using the freeze-thaw method and then transformed into wild-type (Col) plants. For selection of transgenic plants, seeds were sterilized and germinated on MS medium containing $25 \mathrm{mg} / \mathrm{L}$ hygromycin. Seedlings exhibiting resistance to hygromycin were transplanted and grown in a greenhouse at $22^{\circ} \mathrm{C}$ under a $16-\mathrm{h} / 8$-h light/dark regime. Seeds from each transgenic plant were harvested separately for subsequent observation.

CRISPR/Cas9 plasmid construction and mutant screening. The 23-bp guide RNA sequences (including PAM) were selected within the target genes, and their targeting specificity was confirmed by a Blast search against the Arabidopsis genome (https://www.arabidopsis.org/index.jsp). The designed targeting sequences were synthesized and annealed to form oligo adaptors ${ }^{77}$. The vector psgR-Cas9-At was digested with BbsI and purified using a gel extraction kit. Ligation was carried out in $10-\mu \mathrm{L}$ reaction volumes containing $10 \mathrm{ng}$ of digested psgR-Cas9-At vector and $0.05 \mathrm{mM}$ oligo adaptor, and the ligated product was directly transformed into E. coli competent cells to produce psgR-Cas9-At-1. The vector psgR-Cas9-At-1 was then digested with EcoRI and HindIII, and the validated psgR-Cas-At-1 fragment was inserted into a pCAMBIA1301 vector. GV3101 was selected for Arabidopsis genetic transformation.

Genomic DNA was extracted from $\mathrm{T}_{2}$ transgenic lines, and the primers flanking the designated target site were used for PCR amplification. The PCR products were sequenced and Blasted to identify mutation sites.

RNA extraction and transcript analysis. Total RNA was extracted using Trizol reagent (Invitrogen) from seedlings in MS medium or rosette leaves grown in soil. cDNA was synthesized in 20- $\mu \mathrm{L}$ reaction volumes using $1 \mu \mathrm{g}$ DNase-I-treated total RNA and oligo-dT primers or gene/miRNA-specific primers. Real-time PCR was performed in $20-\mu \mathrm{L}$ volumes containing $3 \mu \mathrm{L}$ of 10 -fold diluted cDNA, $10 \mu \mathrm{L}$ of iQ SYBR Green Supermix (Bio-Rad, Hercules, CA, USA), and $0.25 \mu \mathrm{M}$ of each primer. The analysis was performed in a MyiQ2 Two-color Real-time PCR Detection system (Bio-Rad) using the following cycling conditions: initial denaturation at $95^{\circ} \mathrm{C}$ for $3 \mathrm{~min}$, followed by $45 \mathrm{cycles}$ of $95^{\circ} \mathrm{C}$ for $10 \mathrm{~s}, 55^{\circ} \mathrm{C}$ for $20 \mathrm{~s}$, and $72^{\circ} \mathrm{C}$ for $20 \mathrm{~s}$. The primers used are listed in Supplementary Data 1 . All data were normalized against expression levels of the ACTIN2 gene or 18 S rRNA.

Northern blotting. Total RNA (16-30 $\mu \mathrm{g})$ was separated on $19 \%$ polyacrylamide denaturing gels. The RNA was then transferred to a Hybond membrane (Amersham Biosciences, GE Healthcare) for $2 \mathrm{~h}$ at $200 \mathrm{~mA}$. After crosslinking for $5 \mathrm{~min}$ with UV irradiation, the Hybond membrane was hybridized overnight at $42^{\circ} \mathrm{C}$ with $3^{\prime}$ biotin-marked DNA probes complementary to the predicted miRNA or U6 sequences. Autoradiography of the membrane was performed using a Chemiluminescent Nucleic Acid Detection Module kit ${ }^{78}$.

GUS staining. GUS staining was performed on 14-d-old plants. Seedlings of the transgenic plants were immersed in GUS staining solution (10 mM EDTA, $0.1 \%$ TritonX-100, $100 \mathrm{mM} \mathrm{NaPO}_{4}$ [pH 7.0], $1 \mathrm{mM}$ 5-bromo-4-chloro-3-indolyl glucuronide, $0.5 \mathrm{mM} \mathrm{K}_{3}\left[\mathrm{Fe}(\mathrm{CN})_{6}\right]$, and $\left.0.5 \mathrm{mM} \mathrm{K}_{4}\left[\mathrm{Fe}(\mathrm{CN})_{6}\right]\right)$ and incubated at $37^{\circ} \mathrm{C}$ overnight. Seedlings were then washed with $50 \%$ alcohol and fixed in water for further analysis ${ }^{79}$.

3,3'-Diaminobenzidine tetrahydrochloride (DAB) staining. To detect $\mathrm{H}_{2} \mathrm{O}_{2}$ accumulation, 19 -d-old soil-grown plants were subjected to $22^{\circ} \mathrm{C}$ (control) or $38^{\circ} \mathrm{C}$ (heat treatment) for $1 \mathrm{~h}$. Detached leaves of plants sharing the same pot used for superoxide detection were vacuum-infiltrated with $1 \mathrm{mg} / \mathrm{mL}$ DAB in $1 \mathrm{M}$ Tris-acetate buffer ( $\mathrm{pH}$ 7.5). After incubating samples for $19 \mathrm{~h}$ at room temperature in darkness, chlorophyll was removed using $70 \%$ ethanol.

Rapid amplification of cDNA ends (RACE). Sequence information for AtMIR398b/c and their cis-NATs was retrieved from http://www.arabidopsis.org/. RNA samples were isolated from 3-week-old seedlings. After synthesis of cDNA using a SMARTer RACE 5/3' kit (Clontech), the $5^{\prime}$ and $3^{\prime}$ ends of pri-miR398b/c and their cis-NATs were amplified by RACE-PCR using gene-specific primers (Supplementary Data 1). The $3^{\prime}$ and $5^{\prime}$ PCR products were excised from the gel and cloned into a pMD18T vector (Takara). At least 20 positive colonies were sequenced for each RNA sample.

Transient transformation assay. Four-week-old tobacco leaves were inoculated with Agrobacterium tumefaciens GV3101 containing MIR398a, MIR398b, MIR398c, NAT398b, or NAT398c vectors. Three days later, RNA was extracted from the leaves for qRT-PCR.

Ribonuclease protection assay. RNA was treated with ribonuclease $\mathrm{A}+\mathrm{T}$ at $37^{\circ} \mathrm{C}$ for $30 \mathrm{~min}$, followed by treatment with proteinase $\mathrm{K}$ at $37^{\circ} \mathrm{C}$ for $30 \mathrm{~min}$ and then RNA phenol/chloroform extraction. A negative-control RNA was subjected to the same treatment. cDNA was synthesized and real-time PCR was carried out using different primers designed to target overlapping and nonoverlapping regions of sense and antisense transcripts.

Analysis of NAT398b/c transcriptional activity. Intron-specific and oligo-dT primers were used for reverse transcription following the method of Liu et al. ${ }^{80}$. We then applied intron-specific primers for detection of primary mRNA levels of 
$N A T 398 b / c$ genes in transgenic plants overexpressing MIR398b and MIR398c genes.

Chromatin immunoprecipitation (ChIP) assays. Seedlings of 3-week-old control and transgenic plants were fixed in $1 \%$ formaldehyde under vacuum. Fixed tissues were homogenized, and chromatin was isolated and sonicated. The solubilized chromatin was immunoprecipitated by adding Ser5P Pol II (ab5131, lot GR1713927; Abcam, Cambridge, MA, USA) antibody for overnight incubation at $4{ }^{\circ} \mathrm{C}^{74}$. The amount of precipitated DNA was calculated relative to the total input of chromatin and expressed as a percentage of the total according to the following formula: \% input $=2^{\Delta \mathrm{Ct}} \times 100 \%$, where $\Delta \mathrm{Ct}=\mathrm{Ct}$ (input) $-\mathrm{Ct}$ (IP), and $\mathrm{Ct}$ is the mean threshold cycle of the corresponding amplification reaction. The primers used are listed in Supplementary Data 1.

RNA decay assay. Two-week-old seedlings of Col and p35S::NAT398b/c were treated with $100 \mu \mathrm{g} / \mathrm{mL}$ actinomycin D (Sigma-Aldrich). Materials were harvested after 2 and $8 \mathrm{~h}$. Total RNA was extracted and used for qRT-PCR assays.

Thermotolerance assay. To measure the survival rate of flowers under heat stress, 21-d-old soil-grown plants were subjected to $38^{\circ} \mathrm{C}$ for $3 \mathrm{~d}$, and the number of surviving flowers was recorded. To measure leaf thermotolerance, leaves detached from 16-d-old soil-grown plants were subjected to heat stress for 0 (control) or $7 \mathrm{~h}$ at $38^{\circ} \mathrm{C}$. The treated leaves were then photographed, and the number of green leaves was recorded $5 \mathrm{~d}$ later.

Reporting summary. Further information on research design is available in the Nature Research Reporting Summary linked to this article.

\section{Data availability}

The data that support the findings of this study are available within the paper and its supplementary information. RNA-seq data associated with this study have been deposited in the NCBI SRA under accession PRJNA665283. Source data are provided with this paper. Any other supporting data are available from the corresponding author upon request.

Received: 8 January 2020; Accepted: 30 September 2020;

Published online: 22 October 2020

\section{References}

1. Bartel, D. P. MicroRNAs: genomics, biogenesis, mechanism, and function. Cell 116, 281-297 (2004).

2. Kurihara, Y. \& Watanabe, Y. Arabidopsis micro-RNA biogenesis through Dicer-like 1 protein functions. Proc. Natl Acad. Sci. USA 101, 12753-12758 (2004).

3. Dong, Z., Han, M. H. \& Fedoroff, N. The RNA-binding proteins HYL1 and SE promote accurate in vitro processing of pri-miRNA by DCL1. Proc. Natl Acad. Sci. USA 105, 9970-9975 (2008).

4. Han, M. H., Goud, S., Song, L. \& Fedoroff, N. The Arabidopsis doublestranded RNA-binding protein HYL1 plays a role in microRNA-mediated gene regulation. Proc. Natl Acad. Sci. USA 101, 1093-1098 (2004).

5. Kurihara, Y., Takashi, Y. \& Watanabe, Y. The interaction between DCL1 and HYL1 is important for efficient and precise processing of pri-miRNA in plant microRNA biogenesis. Rna-a Publication of the Rna. Society 12, 206-212 (2006).

6. Fang, Y. D. \& Spector, D. L. Identification of nuclear dicing bodies containing proteins for microRNA biogenesis in living Arabidopsis plants. Curr. Biol. 17, 818-823 (2007).

7. Yang, L., Liu, Z. Q., Lu, F., Dong, A. W. \& Huang, H. SERRATE is a novel nuclear regulator in primary microRNA processing in Arabidopsis. Plant J. 47, 841-850 (2006).

8. Jones-Rhoades, M. W., Bartel, D. P. \& Bartel, B. MicroRNAs and their regulatory roles in plants. Annu. Rev. Plant Biol. 57, 19-53 (2006).

9. Vazquez, F., Gasciolli, V., Crete, P. \& Vaucheret, H. The nuclear dsRNA binding protein HYL1 is required for MicroRNA accumulation and plant development, but not posttranscriptional transgene silencing. Curr. Biol. 14, 346-351 (2004).

10. Carrington, J. C. \& Ambros, V. Role of microRNAs in plant and animal development. Science 301, 336-338 (2003).

11. Kis, A. et al. Polycistronic artificial miRNA-mediated resistance to Wheat dwarf virus in barley is highly efficient at low temperature. Mol. Plant Pathol. 17, 427-437 (2016).

12. Schwab, R., Ossowski, S., Riester, M., Warthmann, N. \& Weigel, D. Highly specific gene silencing by artificial microRNAs in Arabidopsis. Plant Cell 18, 1121-1133 (2006).
13. Sunkar, R., Kapoor, A. \& Zhu, J. K. Posttranscriptional induction of two Cu/ $\mathrm{Zn}$ superoxide dismutase genes in Arabidopsis is mediated by downregulation of miR398 and important for oxidative stress tolerance. Plant Cell 18, 2051-2065 (2006)

14. Lu, T. et al. Strand-specific RNA-seq reveals widespread occurrence of novel cis-natural antisense transcripts in rice. BMC Genomics 13, 721 (2012).

15. Wang, X. J., Gaasterland, T. \& Chua, N. H. Genome-wide prediction and identification of cis-natural antisense transcripts in Arabidopsis thaliana. Genome Biol. 6, R30 (2005).

16. $\mathrm{Yu}, \mathrm{X}$. et al. Global analysis of cis-natural antisense transcripts and their heatresponsive nat-siRNAs in Brassica rapa. BMC Plant Biol. 13, 208 (2013)

17. Kindgren, P., Ivanov, M. \& Marquardt, S. Native elongation transcript sequencing reveals temperature dependent dynamics of nascent RNAPII transcription in Arabidopsis. Nucleic Acids Res. 48, 2332-2347 (2020).

18. Bouchard, J., Oliver, C. \& Harrison, P. M. The distribution and evolution of Arabidopsis thaliana cis natural antisense transcripts. BMC Genomics 16, 444 (2015).

19. Zhang, Y., Liu, X. S., Liu, Q. R. \& Wei, L. Genome-wide in silico identification and analysis of cis natural antisense transcripts (cis-NATs) in ten species. Nucleic Acids Res. 34, 3465-3475 (2006).

20. Lapidot, M. \& Pilpel, Y. Genome-wide natural antisense transcription: coupling its regulation to its different regulatory mechanisms. EMBO Rep. 7, 1216-1222 (2006).

21. Wilusz, J. E., Sunwoo, H. \& Spector, D. L. Long noncoding RNAs: functional surprises from the RNA world. Genes Dev. 23, 1494-1504 (2009).

22. Hatzoglou, A. et al. Natural antisense RNA inhibits the expression of BCMA, a tumour necrosis factor receptor homologue. BMC Mol. Biol. 3, 4 (2002).

23. Faghihi, M. A. et al. Expression of a noncoding RNA is elevated in Alzheimer's disease and drives rapid feed-forward regulation of beta-secretase. Nat. Med. 14, 723-730 (2008).

24. Georg, J. et al. Evidence for a major role of antisense RNAs in cyanobacterial gene regulation. Mol. Syst. Biol. 5, 305 (2009).

25. Munroe, S. H. \& Lazar, M. A. Inhibition of c-erbA mRNA splicing by a naturally occurring antisense RNA. J. Biol. Chem. 266, 22083-22086 (1991).

26. Sureau, A. et al. Characterization of multiple alternative RNAs resulting from antisense transcription of the PR264/SC35 splicing factor gene. Nucleic Acids Res. 25, 4513-4522 (1997).

27. Kim, D. D. et al. Widespread RNA editing of embedded alu elements in the human transcriptome. Genome Res. 14, 1719-1725 (2004).

28. Peters, N. T., Rohrbach, J. A., Zalewski, B. A., Byrkett, C. M. \& Vaughn, J. C. RNA editing and regulation of Drosophila $4 \mathrm{f}$-rnp expression by sas-10 antisense readthrough mRNA transcripts. RNA 9, 698-710 (2003).

29. Lewis, A. et al. Imprinting on distal chromosome 7 in the placenta involves repressive histone methylation independent of DNA methylation. Nat. Genet. 36, 1291-1295 (2004)

30. Tufarelli, C. et al. Transcription of antisense RNA leading to gene silencing and methylation as a novel cause of human genetic disease. Nat. Genet. 34, 157-165 (2003).

31. Zhao, X. et al. Global identification of Arabidopsis lncRNAs reveals the regulation of MAF4 by a natural antisense RNA. Nat. Commun. 9, 5056 (2018).

32. Liu, F., Marquardt, S., Lister, C., Swiezewski, S. \& Dean, C. Targeted 3' processing of antisense transcripts triggers Arabidopsis FLC chromatin silencing. Science 327, 94-97 (2010).

33. Borsani, O., Zhu, J., Verslues, P. E., Sunkar, R. \& Zhu, J. K. Endogenous siRNAs derived from a pair of natural cis-antisense transcripts regulate salt tolerance in Arabidopsis. Cell 123, 1279-1291 (2005).

34. Katiyar-Agarwal, S. et al. A pathogen-inducible endogenous siRNA in plant immunity. Proc. Natl Acad. Sci. USA 103, 18002-18007 (2006).

35. Jabnoune, $M$. et al. A rice cis-natural antisense RNA acts as a translational enhancer for its cognate mRNA and contributes to phosphate homeostasis and plant fitness. Plant Cell 25, 4166-4182 (2013).

36. Deforges, J. et al. Control of cognate sense mRNA translation by cis-natural antisense RNAs. Plant Physiol. 180, 305-322 (2019).

37. Luo, Q. J. et al. Evidence for antisense transcription associated with microRNA target mRNAs in Arabidopsis. PLoS Genet. 5, e1000457 (2009).

38. Zhang, X. M. et al. Genome-wide analysis of plant nat-siRNAs reveals insights into their distribution, biogenesis and function. Genome Biol. 13, R20 (2012).

39. Jiang, N. et al. Sly-lncRNA15492 interacts with Sly-miR482a and affects Solanum lycopersicum immunity against Phytophthora infestans. Plant J. 103, 1561-1574 (2020).

40. Sunkar, R. \& Zhu, J. K. Novel and stress-regulated microRNAs and other small RNAs from Arabidopsis. Plant Cell 16, 2001-2019 (2004).

41. Xu, W. H., Meng, Y. \& Wise, R. P. Mla- and Rom1-mediated control of microRNA398 and chloroplast copper/zinc superoxide dismutase regulates cell death in response to the barley powdery mildew fungus. N. Phytol. 201, 1396-1412 (2014) 
42. Guan, Q. M., Lu, X. Y., Zeng, H. T., Zhang, Y. Y. \& Zhu, J. H. Heat stress induction of miR398 triggers a regulatory loop that is critical for thermotolerance in Arabidopsis. Plant J. 74, 840-851 (2013).

43. Jovanovic, Z., Stanisavljevic, N., Mikic, A., Radovic, S. \& Maksimovic, V. Water deficit down-regulates miR398 and miR408 in pea (Pisum sativum L.). Plant Physiol. Biochem. 83, 26-31 (2014).

44. Jia, X. Y. et al. Differential and dynamic regulation of miR398 in response to ABA and salt stress in Populus tremula and Arabidopsis thaliana. Plant Mol. Biol. 71, 51-59 (2009).

45. Dugas, D. V. \& Bartel, B. Sucrose induction of Arabidopsis miR398 represses two $\mathrm{Cu} / \mathrm{Zn}$ superoxide dismutases. Plant Mol. Biol. 67, 403-417 (2008).

46. Mittler, R. Abiotic stress, the field environment and stress combination. Trends Plant Sci. 11, 15-19 (2006).

47. Schulze, W. X., Schneider, T., Starck, S., Martinoia, E. \& Trentmann, O. Cold acclimation induces changes in Arabidopsis tonoplast protein abundance and activity and alters phosphorylation of tonoplast monosaccharide transporters. Plant J. 69, 529-541 (2012).

48. Xie, Z. et al. Expression of Arabidopsis MIRNA genes. Plant Physiol. 138, 2145-2154 (2005).

49. Yuan, S. X. et al. Antisense long non-coding RNA PCNA-AS1 promotes tumor growth by regulating proliferating cell nuclear antigen in hepatocellular carcinoma. Cancer Lett. 349, 87-94 (2014).

50. Johnsson, P. et al. A pseudogene long-noncoding-RNA network regulates PTEN transcription and translation in human cells. Nat. Struct. Mol. Biol. 20, 440-446 (2013).

51. Ogawa, Y., Sun, B. K. \& Lee, J. T. Intersection of the RNA interference and Xinactivation pathways. Science 320, 1336-1341 (2008).

52. Wu, Z., Fang, X. F., Zhu, D. L. \& Deati, C. Autonomous pathway: FLOWERING LOCUS $C$ repression through an antisense-mediated chromatin-silencing mechanism. Plant Physiol. 182, 27-37 (2020).

53. Kindgren, P., Ard, R., Ivanov, M. \& Marquardt, S. Transcriptional readthrough of the long non-coding RNA SVALKA governs plant cold acclimation. Nat. Commun. 9, 4561 (2018).

54. Volkov, R. A., Panchuk, I. I., Mullineaux, P. M. \& Schoffl, F. Heat stressinduced $\mathrm{H}(2) \mathrm{O}(2)$ is required for effective expression of heat shock genes in Arabidopsis. Plant Mol. Biol. 61, 733-746 (2006).

55. Vandenabeele, $\mathrm{S}$. et al. A comprehensive analysis of hydrogen peroxideinduced gene expression in tobacco. Proc. Natl Acad. Sci. USA 100, 16113-16118 (2003)

56. $\mathrm{Li}, \mathrm{Y}$. et al. Osa-miR398b boosts $\mathrm{H} 2 \mathrm{O} 2$ production and rice blast diseaseresistance via multiple superoxide dismutases. N. Phytol. 222, 1507-1522 (2019).

57. Vanhee-Brossollet, C. \& Vaquero, C. Do natural antisense transcripts make sense in eukaryotes? Gene 211, 1-9 (1998).

58. Henriques, R. et al. The antiphasic regulatory module comprising CDF5 and its antisense RNA FLORE links the circadian clock to photoperiodic flowering. N. Phytol. 216, 854-867 (2017).

59. Wang, H. et al. Genome-wide identification of long noncoding natural antisense transcripts and their responses to light in Arabidopsis. Genome Res. 24, 444-453 (2014)

60. Ietswaart, R., Wu, Z. \& Dean, C. Flowering time control: another window to the connection between antisense RNA and chromatin. Trends Genet. 28 445-453 (2012).

61. Zhang, Y. C. et al. Genome-wide screening and functional analysis identify a large number of long noncoding RNAs involved in the sexual reproduction of rice. Genome Biol. 15, 512 (2014).

62. Di, C. et al. Characterization of stress-responsive lncRNAs in Arabidopsis thaliana by integrating expression, epigenetic and structural features. Plant J. 80, 848-861 (2014)

63. Swiezewski, S., Liu, F., Magusin, A. \& Dean, C. Cold-induced silencing by long antisense transcripts of an Arabidopsis Polycomb target. Nature 462, 799-802 (2009).

64. Palatnik, J. F. et al. Control of leaf morphogenesis by microRNAs. Nature 425, 257-263 (2003).

65. Chen, X. M. A microRNA as a translational repressor of APETALA2 in Arabidopsis flower development. Science 303, 2022-2025 (2004).

66. $\mathrm{Wu}, \mathrm{Z}$. et al. Quantitative regulation of FLC via coordinated transcriptional initiation and elongation. Proc. Natl Acad. Sci. USA 113, 218-223 (2016).

67. Wang, K. C. et al. A long noncoding RNA maintains active chromatin to coordinate homeotic gene expression. Nature 472, 120-124 (2011).

68. Gomez, J. A. et al. The NeST long ncRNA controls microbial susceptibility and epigenetic activation of the interferon-gamma locus. Cell 152, 743-754 (2013).

69. Wang, Y. et al. Overexpressing lncRNA LAIR increases grain yield and regulates neighbouring gene cluster expression in rice. Nat. Commun. 9, 3516 (2018).
70. Luo, S. et al. Divergent lncRNAs regulate gene expression and lineage differentiation in pluripotent cells. Cell Stem Cell 18, 637-652 (2016).

71. Wahid, A., Gelani, S., Ashraf, M. \& Foolad, M. R. Heat tolerance in plants: an overview. Environ. Exp. Bot. 61, 199-223 (2007).

72. Chen, $K$. et al. Translational regulation of plant response to high temperature by a dual function tRNAHis guanylyltransferase in rice. Mol. Plant 12, 1123-1142 (2019)

73. Feder, M. E. \& Hofmann, G. E. Heat-shock proteins, molecular chaperones, and the stress response: evolutionary and ecological physiology. Annu. Rev. Physiol. 61, 243-282 (1999).

74. Li, S. et al. HEAT-INDUCED TAS1 TARGET1 mediates thermotolerance via HEAT STRESS TRANSCRIPTION FACTOR Ala-directed pathways in Arabidopsis. Plant Cell 26, 1764-1780 (2014).

75. Chopin, F. et al. The Arabidopsis ATNRT2.7 nitrate transporter controls nitrate content in seeds. Plant Cell 19, 1590-1602 (2007).

76. David, L. C. et al. Proanthocyanidin oxidation of Arabidopsis seeds is altered in mutant of the high-affinity nitrate transporter NRT2.7. J. Exp. Bot. 65, 885-893 (2014).

77. Liu, W. S. et al. A detailed procedure for CRISPR/Cas9-mediated gene editing in Arabidopsis thaliana. Sci. Bull. 60, 1332-1347 (2015).

78. Liu, Z. Y., Jia, L. G., Wang, H. \& He, Y. K. HYL1 regulates the balance between adaxial and abaxial identity for leaf flattening via miRNA-mediated pathways. J. Exp. Bot. 62, 4367-4381 (2011).

79. Yu, L., Yu, X., Shen, R. \& He, Y. HYL1 gene maintains venation and polarity of leaves. Planta 221, 231-242 (2005).

80. Liu, F. et al. The Arabidopsis RNA-binding protein FCA requires a lysinespecific demethylase 1 homolog to downregulate FLC. Mol. Cell 28, 398-407 (2007).

\section{Acknowledgements}

This work was supported by the National Programs for Science and Technology Development of China (Grant no. 2016YFD0101900) and the Natural Science Foundation of China (Grant nos. 31471883 and 31571261).

\section{Author contributions}

Y.L. and J.Y. conceived the project; Y.L., J.Y., and Y.H. designed the experiments; Y.L. performed the experiments; Y.L., Y.H., and X.L. analyzed and interpreted the data; Y.L., J.Y., and Y.H. wrote the manuscript.

\section{Competing interests}

The authors declare no competing interests.

\section{Additional information}

Supplementary information is available for this paper at https://doi.org/10.1038/s41467 020-19186-x.

Correspondence and requests for materials should be addressed to J.Y. or Y.H.

Peer review information Nature Communications thanks Sebastian Marquardt and the other, anonymous reviewers for their contribution to the peer review of this work. Peer review reports are available.

Reprints and permission information is available at http://www.nature.com/reprints

Publisher's note Springer Nature remains neutral with regard to jurisdictional claims in published maps and institutional affiliations.

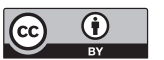

Open Access This article is licensed under a Creative Commons Attribution 4.0 International License, which permits use, sharing, adaptation, distribution and reproduction in any medium or format, as long as you give appropriate credit to the original author(s) and the source, provide a link to the Creative Commons license, and indicate if changes were made. The images or other third party material in this article are included in the article's Creative Commons license, unless indicated otherwise in a credit line to the material. If material is not included in the article's Creative Commons license and your intended use is not permitted by statutory regulation or exceeds the permitted use, you will need to obtain permission directly from the copyright holder. To view a copy of this license, visit http://creativecommons.org/ licenses/by/4.0/.

(C) The Author(s) 2020 